Illinois State University

ISU ReD: Research and eData

Theses and Dissertations

7-22-2015

\title{
Does Simulation Improve Nursing Student Knowledge, Skills, and Attitudes In Identifying Delirium Superimposed on Dementia?
}

Sheryl A. Kelly

Illinois State University, skelly2@ilstu.edu

Follow this and additional works at: https://ir.library.illinoisstate.edu/etd

Part of the Education Commons, and the Nursing Commons

\section{Recommended Citation}

Kelly, Sheryl A., "Does Simulation Improve Nursing Student Knowledge, Skills, and Attitudes In Identifying Delirium Superimposed on Dementia?" (2015). Theses and Dissertations. 456.

https://ir.library.illinoisstate.edu/etd/456

This Dissertation is brought to you for free and open access by ISU ReD: Research and eData. It has been accepted for inclusion in Theses and Dissertations by an authorized administrator of ISU ReD: Research and eData. For more information, please contact ISUReD@ilstu.edu. 


\title{
DOES SIMULATION IMPROVE NURSING STUDENT KNOWLEDGE, SKILLS, AND ATTITUDES IN IDENTIFYING DELIRIUM SUPERIMPOSED ON DEMENTIA?
}

\author{
Sheryl A. Kelly
}

66 Pages

This dissertation is comprised of three manuscripts, to be submitted for publication, exploring the use of simulation in geriatric nursing education. The first manuscript is a literature review. Although, a number of articles exist on the use of simulation in nursing education, there is a paucity of research using simulation emphasizing the care of patients with dementia and delirium as a pedagogical strategy or those using a stringent research design.

The second manuscript provides an overview of two conceptual frameworks (National League for Nursing (NLN)/Jeffries Simulation Framework and situated cognition learning framework) and recommends merging them to guide researchers as they explore application and transfer of learning from simulations to clinical settings.

The third manuscript outlines the method and results of a quasi-experimental study to determine if undergraduate nursing students' knowledge, skills, and attitudes improve following a simulation experience. Twenty-six senior undergraduate nursing students in their last semester gerontological nursing course participated in the study. 
Both groups received didactic content and the experimental group also participated in a simulation on care of the older adult with dementia and delirium. To examine differences in knowledge and attitudes all students completed a pretest prior to the scheduled simulation day and posttest one month later. To examine skills, students viewed a videotaped encounter of a patient with delirium/dementia and completed the Confusion Assessment Method (CAM) on the posttest. The experimental group maintained their knowledge scores while the control group scores dropped. Attitude scores were higher for those in the experimental group and all students correctly identified delirium superimposed on dementia using the CAM.

KEYWORDS: Application of learning, Delirium, Dementia, NLN/Jeffries Framework, Nursing Education, Simulation, Situated Cognition, Transfer of knowledge 


\title{
DOES SIMULATION IMPROVE NURSING STUDENT KNOWLEDGE, SKILLS, AND ATTITUDES IN IDENTIFYING DELIRIUM SUPERIMPOSED ON DEMENTIA?
}

\author{
SHERYL A. KELLY
}

A Dissertation Submitted in Partial Fulfillment of the Requirements for the Degree of

DOCTOR OF PHILOSOPHY

Mennonite College of Nursing

ILLINOIS STATE UNIVERSITY 
Copyright 2015 Sheryl A. Kelly 
DOES SIMULATION IMPROVE NURSING STUDENT KNOWLEDGE, SKILLS, AND ATTITUDES IN IDENTIFYING DELIRIUM SUPERIMPOSED ON DEMENTIA?

SHERYL A. KELLY

COMMITTEE MEMBERS:

Susan P. Kossman, Chair

Mary J. Dyck

Kim S. Astroth

Susan G. Forneris 


\section{ACKNOWLEDGMENTS}

I dedicate this dissertation to my husband, Scott and children, Bryan and Sarah. I could not have made this journey without your love, encouraging words and support. I am grateful for the understanding you showed when I was unable to attend an event or gathering. Thank you for all the hugs and chocolate. Thank you to my Mother and close friends for their love and support.

I would like to thank my committee members, Drs. Susan Kossman, Mary Dyck, Kim Astroth, and Susan Forneris, for their expertise and guidance throughout this process. A special thank you to my committee chair Dr. Susan Kossman for supporting me every step of the way by picking me up every time I hit bottom and gently pushing me along. I made it!

Lastly, I would like to thank the faculty, staff, and my classmates at Mennonite College of Nursing. I am grateful for your guidance and support throughout my $\mathrm{PhD}$ program. Your smiles and encouraging words kept me going. 


\section{CONTENTS}

\section{Page}

ACKNOWLEDGMENTS

CONTENTS $\quad$ ii

TABLES $\quad$ iv

FIGURES

CHAPTER

I. SIMULATION IN GERIATRIC NURSING EDUCATION:

A LITERATURE REVIEW 1

Abstract 1

Introduction and Purpose $\quad 2$

Background and Significance $\quad 3$

Dementia $\quad 3$

Delirium 3

Nursing Care of the Hospitalized Patient with Dementia 4

Simulation as an Educational Strategy 5

Use of Simulation to Improve Nursing Care of Elders with

Dementia 8

Method 9

Results 9

Discussion 13

Conclusion 16

References $\quad 17$

$\begin{array}{ll}\text { Figures } & 20\end{array}$ 
II. SITUATED COGNITION, THE NLN/JEFFRIES SIMULATION FRAMEWORK, AND APPLICATION OF LEARNING

Abstract

Introduction and Purpose

NLN/Jeffries Simulation Framework

Situated Cognition

Merging the Models

References

Figures

Appendix A

III. DOES SIMULATION IMPROVE NURSING STUDENT

KNOWLEDGE, SKILLS, AND ATTITUDES IN IDENTIFYING DELIRIUM SUPERIMPOSED ON DEMENTIA?

Abstract

Introduction

40

Purpose and Aims

Framework

Method

Research Design

Sample and Setting

Variables and Instruments

Procedure

Data Analysis

Results

Specific Aim 1

Specific Aim 2

Discussion

Limitations

Conclusion

References

59

Figures

63

Tables 


\section{TABLES}

Table $\quad$ Page

1. Variables, Operational Definitions, and Instruments 64

2. Specific Aim 1 Results 66 


\section{FIGURES}

Figure $\quad$ Page

1. Review of Literature Flowchart 20

2. The NLN/Jeffries Simulation Framework 36

3. Combination of the Situated Cognition Learning Framework and the NLN/Jeffries Simulation Framework

4. Combination of the Situated Cognition Learning Framework and the NLN/Jeffries Simulation Framework 


\title{
CHAPTER I
}

\section{SIMULATION IN GERIATRIC NURSING EDUCATION: A LITERATURE REVIEW}

Chapter 1 is a manuscript that explores the literature on the use of simulation as an educational intervention to improve nursing care of the hospitalized older adult with dementia and identify student outcomes associated with nursing care of this population. The manuscript will be submitted to the Journal of Nursing Education for publication.

\begin{abstract}
Background: Nursing educators are challenged with finding effective learning experiences that highlight the knowledge, skills, and attitudes needed to provide quality care for cognitively impaired individuals. Simulations are a teaching strategy with the potential to address this need.

Method: A literature search was conducted to explore the state of science on use of simulation as an educational intervention to improve nursing care and identify student outcomes associated with nursing care of older adults with dementia.

Results: Search results identified 352 articles; after reviewing all articles' abstracts for relevancy; and reading those selected for further review, five fully met criteria.

Conclusion: Research using rigorous design and valid and reliable measures is limited on the use of simulation in clinical or undergraduate nursing education focusing on care of the older adult.
\end{abstract}




\section{Introduction and Purpose}

Nurses face many challenges caring for an aging population with dementia (Moyle, Olorenshaw, Wallis, \& Borbasi, 2008). Specialized knowledge, skills, and attitudes are needed to provide quality care and improve outcomes for frail and cognitively impaired individuals in the acute care setting (Moyle et al., 2008). Scarcity of clinical sites and effective clinical learning experiences to attain these skills pose barriers to many nursing programs (Ironside \& McNelis, 2010). Nurse educators are challenged with developing innovative learning opportunities to engage students and prepare them for the care required of the older adult population (Tagliareni, Cline, Mengel, McLaughlin, \& King, 2012). Simulated experiences are a teaching strategy with the potential to address this gap.

Simulation offers an educational experience that mimics the real world setting (Jeffries, 2005). This innovative technique has shown success in increasing nursing knowledge, skills and attitudes (Beddingfield, Davis, Gilmore, \& Jenkins, 2011; Foronda, Liu, \& Bauman, 2013; Gates, Parr, \& Hughen, 2012; Weaver, 2011). However, there is little evidence about the effectiveness of simulated clinical experiences for improving nursing students' knowledge, skills, and attitudes about care of hospitalized elders with dementia. This paper presents an overview of the issues surrounding the provision of quality care for hospitalized elders with dementia and the use of simulation in nursing education followed by a review of the literature specific to use of simulation to improve nursing students' knowledge, skills and attitudes regarding nursing care of elders with dementia. 


\section{Background and Significance}

\section{Dementia}

Dementia is a neurodegenerative condition identified by impairment in two or more cognitive areas (Grand, Casper, \& MacDonald, 2011). Care for individuals with dementia is challenging as the disease progresses from memory loss to inability to communicate and perform activities of daily living (Grand, Casper, \& MacDonald, 2011). The estimated number of individuals with dementia worldwide is 36.5 million people with rates expected to double every 20 years as the population increases (Prince et al., 2013). Presently, in the United States, almost 4 million people live with dementia (Prince et al., 2013). The prevalence of this debilitating condition is increasing and leading to economic burden as the demand for care increases (Prince, et al., 2013).

\section{Delirium}

Delirium, an acute onset of confusion with a fluctuating course, occurs in many hospitalized elders and leads to poor patient outcomes if unrecognized and untreated (Inouye, Studenski, Tinetti, \& Kuchel, 2007; Wei, Fearing, Sternberg, \& Inouye, 2008). In a review of 425 hospitalized patients admitted with acute hip fracture, $56 \%$ of patients who also had dementia developed delirium during the hospitalization compared to $26 \%$ of patients without dementia (Lee, Mears, Rosenberg, Leoutsakos, Gottschalk, \& Sieber, 2011).

Delirium when superimposed on dementia is challenging for healthcare providers to recognize (Fick, Hodo, Lawrence, \& Inouye, 2007). Fick et al. (2007) found that 83\% of acute care nurses who viewed video vignettes of hospitalized patients with delirium or delirium superimposed on dementia were able to identify dementia, but only $21 \%$ were 
able to identify delirium superimposed on dementia (Fick et al., 2007). The nurses identified changes in patient status within the vignettes but did not recognize the underlying cause of the change as delirium (Fick et al., 2007). Since the number of persons with dementia is increasing, interventions are needed to improve nurse knowledge in the identification and management of patients with dementia and delirium (Fick et al., 2007).

\section{Nursing Care of the Hospitalized Patient with Dementia}

Nursing care of the hospitalized patient with dementia can be challenging, stressful, and frustrating (Baillie, Cox, \& Merritt, 2012; Byers \& France, 2008; Moyle et al., 2008). Challenges in the care of the hospitalized patient with dementia include concerns with consent for treatment, patient safety, good nutrition, appropriate hygiene, and decreasing agitation (Weitzel et al., 2011). Delirium superimposed on dementia adds additional challenges for healthcare providers (Wei et al., 2008). Decreasing agitation and managing aggression are viewed as very time consuming and lead to negative attitudes of the healthcare staff towards providing care (Moyle et al., 2010).

In a phenomenological study, Byers and France (2008) found that acute care nurses felt they lacked time for meeting all the needs of patients with dementia and their caregivers. They noted inappropriate staffing added to stress and led to feelings of frustration from inability to provide quality and safe care (Byers \& France, 2008).

In a review of nursing care practices, Moyle et al. (2008), found nurses lacked knowledge about dementia and held negative attitudes towards aging. Development of staff knowledge in caring for patients with dementia was deemed important in providing safe quality care for this population (Moyle et al., 2008). 
An increasing aging population with many who suffer from dementia warrants further research on quality nursing care and patient safety. Research on nursing interventions to increase nursing knowledge and improve attitudes may subsequently improve nursing care for those with dementia (Moyle et al., 2008).

\section{Simulation as an Educational Strategy}

An intervention using simulation provides an active learning environment (Jeffries, 2005). Simulation in nursing education consists of students performing faculty monitored nursing care on an actor portraying a patient or a mannequin in a learning environment, followed by a facilitator led debriefing to discuss learning objectives and patient outcomes (Jeffries, 2005). In the Bearnson and Wiker (2005) study, nursing students' perceived a gain in knowledge and confidence through active participation in a simulated scenario. A simulation intervention may enhance knowledge and change behaviors thereby improving nursing care related to hospitalized individuals with dementia.

Simulation in clinical education. Simulation is used in nursing staff development and nursing education to increase knowledge, improve skills, and change attitudes to improve nursing care. In a systematic review of the literature on the use of high-fidelity simulation in staff education, Hallenbeck (2012) noted that research has been conducted in the hospital setting using simulation for orientation programs, maternal-child care, critical care, advanced cardiac life support classes, and general staff development on unit specific competencies. The use of simulation in orientation programs increased confidence in general practice and emergencies as well as staff retention (Hallenbeck (2012). In maternal-infant and critical care units, simulation 
improved communication and teamwork, enhanced learning, improved safety, assisted with recruitment, and improved clinical competencies (Hallenbeck, 2012). Hallenbeck (2012) noted that studies on simulation education are limited within the population of practicing nurses and suggested future research should explore the transfer of knowledge gained in simulation to the bedside.

Research is especially limited on the use of simulation for clinical education in geriatrics. Mager, Lange, Greiner, and Saracino (2012) used simulation to evaluate outcomes of the Expanded Learning and Dedication to Elders in the Region (ELDER) project. This three year project focused on educating nurses and nursing assistants on best practices related to care of the elder. In the final year, Mager and colleagues (2012) evaluated the ability of 104 Registered Nurses, Licensed Practical Nurses, and Nursing Assistants from two long-term care facilities and one home care agency to use best practices in patient care and team communication through participation in their roles in three simulation scenarios. The project developed best practice identifier checklists indicated that $97 \%$ to $100 \%$ of the participants could demonstrate appropriate interprofessional communication, teamwork, and provide appropriate care to patients during the simulations (Mager et al., 2012). Participants indicated through a project evaluation survey that learner objectives were met $97 \%$ to $100 \%$ of the time and all the simulation experiences were noted to be useful by $92 \%$ to $100 \%$ of the participants (Mager et al., 2012).

Simulation in undergraduate nursing education. Weaver (2011) conducted an integrative review of the literature published between 1998 and 2008 on high fidelity simulation and nursing education. The 24 reviewed studies indicated that student 
confidence and knowledge increased following simulations using high fidelity simulation (Weaver, 2011). Weaver highlighted the need for future research examining transfer of knowledge to the clinical setting by new nurses and comparing knowledge acquired by nursing students in simulation to knowledge acquired during their clinical rotations.

Foronda, Liu, and Bauman (2013) conducted an integrative review of the literature on simulation in undergraduate nurse education between 2007 and 2012 to determine the state of the science about simulation using mannequins in nursing education. The authors reviewed 101 research articles and identified five themes: increased confidence/self-efficacy, satisfaction with simulation, decreased anxiety/stress, attainment of skills/knowledge, and opportunities for interdisciplinary experiences that improved communication. The authors suggested future research in knowledge and skills retention and transfer to the clinical setting as well as the increased use of reliable, valid evaluation methods and measureable objectives to benefit educators and researchers as they examine these concepts (Foronda et al., 2013).

Beddingfield, Davis, Gilmore, and Jenkins (2011) compared knowledge gained by nursing students in their final semester after participating in two types of patient care experiences: a) care of the postoperative $\mathrm{CABG}$ patient in the clinical setting and b) care of the same type patient using high fidelity simulation. Following the patient care experiences, students were given a critical care exam with results indicating no significant difference between test scores (cognitive gain) and type of patient care experience (Beddingfield et al., 2011). The authors identified student sample size and the reliability and validity of test items as limitations and a need for continued research on measurable learning outcomes (Beddingfield et al., 2011). 
Gates, Parr, and Hughen (2012) examined students' knowledge acquisition through classroom lecture or lecture plus simulation with 104 first year undergraduate nursing students who attended classroom lectures about care of the patients with pulmonary embolism and gastrointestinal bleeding. Students were randomly assigned to participate in a simulation scenario focusing on either pulmonary embolism or gastrointestinal bleed followed by a one hour debriefing then completed a 10 item exam on each topic (Gates, et al., 2012). The exam scores on the pulmonary embolism questions for students participating in the pulmonary embolism scenario were significantly higher than the scores of students participating in the gastrointestinal bleed scenario (Gates, et al., 2012). The same was found with the exam scores on gastrointestinal bleeding questions for students participating in the gastrointestinal bleed scenario compared to those participating in the pulmonary embolism scenario (Gates, et al., 2012). The results indicate that participation in the simulations improved exam scores (Gates, et al., 2012). Limitations to the study included the use of several different clinical instructors to run the scenarios, small sample size, and the possibility students had previously cared for these types of patients in the clinical setting (Gates, et al., 2012). The authors recommend future research on the amount of preparation needed prior to the simulated experience compared to the level of nursing student (Gates et al., 2012).

\section{Use of Simulation to Improve Nursing Care of Elders with Dementia}

We conducted a literature review to explore the state of science about use of simulation as an educational intervention to improve nursing care of the hospitalized older adult with dementia. The specific aims of the review were (1) to explore how simulation is used as an educational intervention in teaching nursing care of the older 
adult with dementia and (2) to identify student outcomes associated with the use of simulation as an educational intervention in teaching nursing care of the of the older adult with dementia.

\section{Method}

A literature search was conducted in the Cumulative Index of Nursing and Allied Health Literature (CINAHL), Cochrane, Joanna Briggs, and PubMed databases using the terms: simulation, nursing, older adult/aged, and dementia. Inclusion criteria for the articles included those written in English and published in nursing peer-reviewed journals within the last 15 years. Articles focusing on other health professionals and the use of simulation as a means other than providing education for care of the older adult were excluded. Search results identified 352 articles; after reviewing all articles' abstracts for relevancy, 25 articles were selected for further review. After reading these articles, five fully met the criteria to explore the use of simulation to improve nursing knowledge, skills, and attitudes in nursing care of older adults with dementia (Figure 1).

\section{Results}

Although a number of articles exist on dementia care outcomes and the use of simulation in nursing education, very few relate to the use of simulation in education on dementia care and none to date focus on the use simulation to improve outcomes for hospitalized patients with dementia. Research is especially limited on the use of simulation in clinical or undergraduate nursing education focusing on care of the older adult.

Johnson et al. (2012), conducted a quantitative quasi-experimental multi-site study on the effect role modeling has on clinical judgment with 275 undergraduate 
nursing students enrolled in their first year clinical course at four nursing schools in the United States $(n=221)$ or the United Kingdom $(n=54)$. Both the treatment and control groups of students received content about nursing care of the surgical patient and participated in a simulation scenario with debriefing that focused on care of the geriatric patient prior to and following surgical repair of a hip fracture and management of delirium. Students assigned to the treatment group also viewed a video of a nurse role modeling the thinking process (voice-over narrative) in the scenario prior to their participation in the simulation. All students completed an author developed 5-point Likert survey. There were no differences between students in the control and treatment groups in the United States; they rated the simulation experience as helpful in identifying and responding to patient symptoms and in improving their confidence in providing nursing care to the patient with delirium. Students in the United Kingdom had highly significant differences $(p=.000)$ between control and treatment groups on satisfaction with the simulation experience especially confidence with care. The authors suggested some of the difference in satisfaction and self-confidence could be related to content and clinical organization in the United Kingdom program. In another aspect of the study, trained observers used the Lasater Clinical Judgment Rubric to evaluate clinical judgment of the team leaders $(\mathrm{n}=94)$ in the simulations. Students in the treatment group had significantly higher scores in noticing, interpreting, and responding $(\mathrm{p}=.000)$ in all schools $(\mathrm{Johnson}$ et al., 2012). Qualitative data collected on transfer of knowledge in this study were resulted in a different manuscript. The researchers identified future areas for research on the effect of clinical judgment and care of the older adult, such as the use of a live versus 
videotaped expert role model in simulation and the use of a role model the same age as students (Johnson et al., 2012).

Lasater, Johnson, Ravert, and Rink (2014) reported qualitative findings of the same Johnson et al., (2012) study and extended it to "explore whether clinical judgment skills transfer to the clinical setting" (p. 257). Qualitative data were collected at two different time points using a similar author developed questionnaire. First, immediately following the simulation on post-surgical care of the geriatric patient, the 275 students identified in the 2012 study ( $\mathrm{n}=221$ United States, $\mathrm{n}=54$ United Kingdom) completed an 11 item reflective questionnaire focusing on clinical judgment. Second, following a clinical rotation four weeks after the simulation, a subset of this group $(\mathrm{n}=134$ United States), completed another survey similar to the first 11 item reflective questionnaire but with an additional question asking students to reflect on whether participation in the postsurgical simulation prepared them for care of the real life post-surgical patient. The researchers identified differences between the treatment and control groups on the themes "knowing what to expect" and "increased confidence" at post simulation (Lasater et al., 2014, p. 261). Few differences were found between treatment and control group four weeks after the simulation. Themes from student's responses at post care included "heightened awareness", "increased confidence", and felt "simulation learning helped them to care for the postoperative patients" (Lasater et al., 2014, p. 262).

Paquette, Bull, Wilson, and Dreyfus (2010) conducted a study using improvisational actors as standardized patients portraying an elder with delirium superimposed on dementia and heart failure in a simulation experience for 100 third year baccalaureate nursing students over two consecutive semesters $(\mathrm{n}=50$ Fall, $\mathrm{n}=50$ 
Spring) of a gerontological nursing course. The simulation scenario focused on interaction with an agitated, disoriented patient who was also short of breath. The enrolled students reported to the simulation lab in groups of 25 and seven students from each group participated in the scenario while the other students observed through real time video. Student participants were invited to complete a faculty developed survey to rate their comfort in talking with confused elders before and after the simulation. Of the 100 students 63 completed the survey ( $\mathrm{n}=35$ Fall, $\mathrm{n}=28$ Spring). Ratings increased from $37.2 \%$ pre- to $68.6 \%$ post-simulation for students in the Fall semester and from $62.1 \%$ pre- to $82.2 \%$ post- simulation for students in the Spring semester (Paquette et al., 2010). Students in the Spring semester were in their second clinical course which may have contributed to their higher comfort ratings both pre and post-simulation (Paquette et al., 2010). The authors discuss the need for additional cues from faculty during the simulation as students concentrated more on communication with the dementia patient and overlooked the physical symptoms of heart failure (Paquette et al., 2010).

Sheets and Ganley (2011) conducted an education day for baccalaureate nursing students that included participation in a simulated scenario of a hospitalized older adult and "active learning stations" one of which included students performing a Mini-Cog and the Confusion Assessment Method (CAM) on each other. Students completed the National League for Nursing (NLN) Simulation Design Scale, Educational Practices Questionnaire and Student Satisfaction and Self-Confidence in Learning Scale. All 5point likert scales results indicated student satisfaction with the education day and improved self-confidence (means on all scales $\geq 4$.37) (Sheets \& Ganley, 2011). The authors suggested future research to measure transfer of learning to the clinical setting. 
One study examined the use of simulation focusing on care of elders with practicing nurses (RNs and LPNs), nurse educators (RNs) and student nurses. Palmer et al. (2008) used an online library of geriatric specific simulations in a continuing education program for nurse educators and practicing nurses. Expert faculty using geriatric nursing competencies from the American Association of Colleges of Nursing $(\mathrm{AACN})$ and the John A. Hartford Foundation Institute for Geriatric Nursing developed these online geriatric scenarios focused on nursing care of elders in long term and acute care settings (Palmer, et al., 2008). During learner leveled workshops, (one day for RNs, LPNs, and nursing students; three days for nurse educators) 312 subjects received didactic nursing content on geriatric conditions and participated with small groups in 4-5 simulated scenarios (Palmer, et al., 2008). Participants completed a 4-item knowledge quiz for each scenario (pretest) and at the end of the workshop (posttest) (Palmer et al., 2008). Additionally, they evaluated the scenarios for relevance to practice and opportunity for skill improvement (Palmer et al., 2008). Participants demonstrated a statistically significant increase in knowledge after the simulation $(62.7 \%$ pretest and $76.7 \%$ posttest, $\mathrm{t}=13.65, \mathrm{p}=.001)$, however the authors noted that scores below $80 \%$ show the need for additional learning (Palmer et al., 2008). Evaluations indicated satisfaction with the workshop and skill attainment during the simulations (Palmer et al., 2008).

\section{Discussion}

While there is an increasing body of research on the use of simulation in nursing and care of the geriatric patient, there remains a paucity of studies on the use of simulation in nursing education related to care of the dementia patient, and those with 
rigorous research designs using objective, reliable, and valid measures. The use of strong designs and robust instruments provides researchers and educators a more accurate evaluation of student learning and leads to more appropriate feedback of student performance (Adamson, Gubrud, Sideras, \& Lasater, 2011). Of 25 studies, only five focused on use of simulation in nursing education related to care of the individual with dementia and reported the methodology, including instruments and data analysis.

Three of the five studies reviewed were quantitative descriptive studies using instruments to determine, report, or describe student outcomes related to the use of simulation in geriatric nursing education (Palmer et al., 2008; Paquette et al., 2010; Sheets \& Ganley, 2011). Johnson et al. (2012) used a quasi-experimental design (part one of a multi-site study) and Lasater et al. (2014) used a qualitative design (part two of the same multi-site study) to determine expert role modeling and explore the transfer of clinical judgment. The results of these five studies indicate nursing students, practicing nurses, and educators found the use of simulation in geriatric nursing education increased learner confidence and knowledge in care of the patient with dementia, and role model expectations; simulations also improved skills specific to geriatric care and attitudes in communicating with the older adult.

These results are consistent with the literature on simulation in nursing education. Researchers found simulation improves nursing skills, knowledge, communication and teamwork when used with practicing nurses (Hallenbeck, 2012; Landry et al., 2006; Mager et al., 2012; \& Merchant, 2012). Nursing students improve exam scores, increase confidence in patient care, improve interdisciplinary communication, and improve skills when nursing programs use simulation as an 
educational strategy (Foronda et al., 2013; Gates et al., 2012; \& Weaver, 2011).

However, limitations of this body of research include a lack of rigorous research design and the use of instruments without adequate reliability and validity testing. These limitations may lead to an inability to measure transfer of knowledge to the clinical setting.

All five of the studies reviewed used a self-report instrument for student's evaluating the simulation learning experience (Johnson et al., 2012; Lasater et al., 2014; Palmer et al., 2008; Paquette et al., 2010; Sheets \& Ganley, 2011). The self-report instrument was researcher developed in four of the studies (Johnson et al., 2012; Lasater et al., 2014; Palmer et al., 2008; Paquette et al., 2010) while, Sheets \& Ganley (2011) used NLN instruments with validity and reliability testing. Faculty used the Lasater Clinical Judgment Rubric in the Johnson et al., (2012) study to rate clinical judgment of the team leaders in the simulations, and Palmer et al., (2008) included a pretest and posttest on knowledge. Although the NLN scales and the Lasater Clinical Judgment Rubric had documented reliability and validity testing, many simulation in nursing education studies used self-report instruments with no reliability and validity testing (Mager et al., 2012; Foronda et al., 2013; Beddingfield et al., 2011) .

The use of self-report and instruments that lack reliability and validity is an obstacle to an accurate evaluation of student learning (Adamson \& Kardong-Edgren, 2011). Educators require research results from evidence based evaluation instruments to make appropriate formative assessments of teaching and learning (Adamson \& KardongEdgren, 2011). A number of tools have been developed for evaluating aspects of simulation in nursing education and have initial validly and reliability, yet they have not 
been widely tested (Adamson \& Kardong-Edgren, 2011). Additional studies using these tools and including analysis of their reliability and validity in that setting may lead to stronger evidence. The use of reliable and valid instruments, objective means of measurement along with self-report, experimental designs, increased sample sizes, and multi-site studies are needed for this important evidence based research to define future education practices. Rigorous research is needed to support the use of simulation in nursing education related to care of the person with dementia.

\section{Conclusion}

With an aging population, nurses provide care to an increasing number of individuals with dementia and delirium. Nursing care of this population is full of challenges and requires specialized knowledge, skills and attitudes. The scarcity of clinical sites influences our nursing education programs. Simulation is a versatile educational intervention that can meet many of the challenges faced in educating nurses to care for persons with dementia. Research shows that knowledge and confidence increase after simulated clinical experiences. Nursing education needs research that includes experimental design and objective valid and reliable measures to address application and transfer of knowledge and bridge the gap between simulation and clinical practice. 


\section{REFERENCES}

Adamson, K. A., Gubrud, P., Sideras, S., \& Lasater, K. (2011). Assessing the reliability, validity, and use of the Lasater Clinical Judgment Rubric: Three approaches. Journal of Nursing Education, 51(2), 66-73.

Adamson, K. A. \& Kardong-Edgren, S. (2011). A method and resources for assessing the reliability of simulation evaluation instruments. Nursing Education Perspectives, 33(5), 334-339.

Baillie, L., Cox, J., \& Merrit, J. (2012). Caring for older people with dementia in hospital part one: Challenges. Nursing Older People, 24(8), 33-37.

Bearnson, C. S. \& Wiker, K. M. (2005). Human patient simulators: A new face in Baccalaureate nursing education at Brigham Young University. Journal of Nursing Education, 44(9), 421- 425.

Beddingfield, S., Davis, B. W., Gilmore, M., \& Jenkins, L. (2011). The effect of highfidelity simulation on examination performance. Teaching and Learning in Nursing, $6,46-49$.

Byers, D. C. \& France, N. (2008). The lived experience of registered nurses providing care to patients with dementia in the acute care setting: A phenomenological study. International Journal for Human Caring, 12(4), 44-49.

Fick, D. M., Hodo, D. M., Lawrence, F., Inouye, S. K. (2007). Recognizing delirium superimposed on dementia: Assessing nurses' knowledge using case vignettes. Journal Gerontological Nursing, 33(2), 40-49.

Foronda, C., Liu, S., Bauman, E. B. (2013). Evaluation of simulation in undergraduate nurse education: An integrative review. Clinical Simulation in Nursing, 10, e409416.

Gates, M. G., Parr, M. B., \& Hughen, J. E. (2012). Enhancing nursing knowledge using high-fidelity simulation. Journal of Nursing Education, 51(1), 9-14.

Grand, J. H., Caspar, S., \& Macdonald, S. W. (2011). Clinical features and multidisciplinary approaches to dementia care. Journal of Multidisciplinary Healthcare, 4, 125-147. 
Hallenbeck, V. J. (2012). Use of high-fidelity simulation for staff education development: A systematic review of the literature. Journal for Nurses in Staff Development, 28(6), 260-269.

Inouye, S. K., Studenski, S., Tinetti, M. E., \& Kuchel, G. A. (2007) Geriatric Syndromes: Clinical, research, and policy implications of a core geriatric concept. Journal of the American Geriatrics Society, 55, 780-791.

Ironside, P. M., McNelis, A. M. (2010). Clinical education in prelicensure nursing programs: Findings from a National Survey. Nursing Education Perspectives. 31(4), 264-265.

Jeffries, P. R. (2005). A Framework for designing, implementing, and evaluating simulations used as teaching strategies in nursing. Nursing Education Perspectives, 26(2), 96-103.

Johnson, E. A., Lasater, K., Hodson-Carlton, K., Siktberg, L., Sideras, S., \& Dillard, N. (2012). Geriatrics in simulation: Role modeling and clinical judgment effect. Nursing Education Perspectives, 33(3), 176-180.

Landry, M., Oberleitner, M. G., Landry, H., \& Borazjani, J. G. (2006). Education and practice collaboration: Using simulation and virtual reality technology to assess continuing nurse competency in the long-term care acute care setting. Journal for Nurses in Staff Development, 22(4), 163-169.

Lasater, K., Johnson, E. A., Ravert, P., \& Rink, D. (2014). Role modeling clinical judgment for an unfolding older adult simulation. Journal of Nursing Education, 53(5), 257-264.

Lee, H. B., Mears, S. C., Rosenberg, P. B., Leoutsakos, J. S., Gottschalk, A., \& Sieber, F. E. (2011). Predisposing Factors for postoperative delirium after hip fracture repair in individuals with and without dementia. Journal of the American Geriatrics Society, 59, 2306-2313.

Mager, D. R., Lange, J. W., Greiner, P. A., \& Saracino, K. H. (2012). Using simulation pedagogy to enhance teamwork and communication in the care of older adults: The ELDER project. The Journal of Continuing Education in Nursing, 43(8), 363-369.

Merchant, D. C. (2012). Does high-fidelity simulation improve clinical outcomes? Journal for Nurses in Staff Development, 28(1), E1-E8.

Moyle, W., Olorenshaw, R., Wallis, M., \& Borbasi, S. (2008). Best practice for the management of older people with dementia in the acute care setting: a review of the literature. International Journal of Older People Nursing, 3, 121-130. 
Moyle, W., Borbasi, S., Wallis, M., Olorenshaw, R., \& Garcia, N. (2010). Acute care management of older people with dementia: a qualitative perspective. Journal of Clinical Nursing, 20, 420-428.

Palmer, M. H,. Kowlowitz, V., Campbell, J., Carr, C., Dillon, R., Durham, C. F., Rasin, J. (2008). Using clinical simulations in geriatric nursing continuing education. Nursing Outlook, 56, 159-166.

Paquette, M., Bull, M., Wilson, S., \& Dreyfus, L. (2010). A complex elder care simulation using improvisational actors. Nurse Educator, 35(6), 254-258.

Prince, M., Bryce, R., Albanese, E., Wimo, A., Ribeiro, W., \& Ferri, C. P. (2013). The global prevalence of dementia: A systematic review and metaanalysis. Alzheimer's \& Dementia, 9, 63-75.

Sheets, I. W. \& Ganley, B. J. (2011). Integrating simulation into a foundational gerontological nursing course. Journal of Nursing Education, 50(12), 689-692.

Tagliareni, M. E., Cline, D. D., Mengel, A., McLaughlin, B. \& King, E. (2012). Quality care for older adults: The NLN advancing care excellence for seniors (ACES) project. Nursing Education Perspectives, 33(3), 144-149.

Weaver, A. (2011). High-fidelity patient simulation in nursing education: An integrative review. Nursing Education Perspectives, 32(1), 37-40.

Wei, L. A., Fearing, M. A., Sternberg, E. J., \& Inouye, S. K. (2008). The confusion assessment method: A systematic review of current usage. Journal of the American Geriatrics Society, 56, 823 - 830.

Weitzel, T., Robinson, S., Barnes, M. R., Berry, T. A., Holmes, J. M., Mercer, S... Kirkbride, G. L. (2011). The special needs of the hospitalized patient with dementia. MedSurg Nursing, 20(1), 13-18. 


\section{FIGURES}

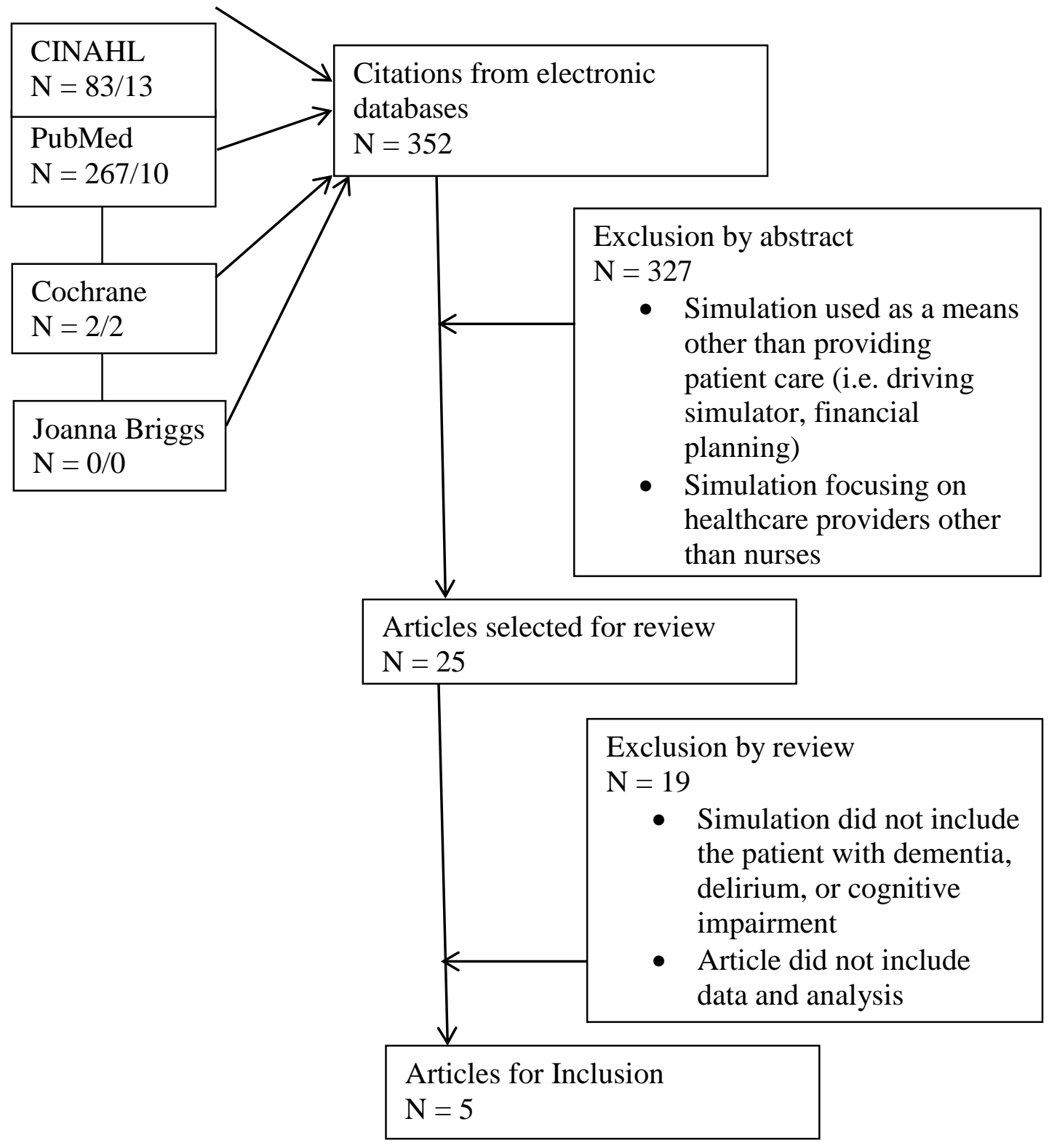

Figure 1. Review of Literature Flowchart. 


\title{
CHAPTER II
}

\section{SITUATED COGNITION, THE NLN/JEFFRIES SIMULATION FRAMEWORK, AND APPLICATION OF LEARNING}

Chapter 2 is a manuscript that provides an overview of two frameworks, NLN/Jeffries Simulation Framework and situated cognition and discusses how merging them supports research to study application of learning in simulation. The manuscript will be submitted to Clinical Simulation in Nursing for publication.

\begin{abstract}
This paper introduces the merging of the NLN/Jeffries Simulation Framework and situated cognition as a conceptual framework for research in simulation design and application of learning. Placing the NLN/Jeffries framework within the framework of situated cognition emphasizes the influence of new concepts, activity and the culture of nursing in all aspects of simulation design and implementation. Strong simulation experiences may increase opportunities for application of student learning from one scenario to another through transformation of learning. The combination of the frameworks and strong simulation experiences may be the bridge for future research in transfer of learning from simulation to the clinical setting.
\end{abstract}




\section{Introduction and Purpose}

The use of simulation as a teaching strategy has become increasingly popular in nursing education. Many nursing programs face challenges finding appropriate clinical sites and simulated clinical experiences that offer students opportunities to improve assessment and psychomotor skills and clinical reasoning in a controlled environment (Ironside \& McNelis, 2010). Well-designed and executed experiences within simulation can be tailored to program outcomes and provide high quality clinical education. Findings from a longitudinal multi-site quasi-experimental study sponsored by the National Council of State Boards of Nursing study showed that well designed and executed simulation can replace up to $50 \%$ of clinical hours (Hayden, Smiley, Alexander, Kardong-Edgren, \& Jeffries, 2014). The National League for Nursing (NLN)/Jeffries Simulation Framework offers a theoretical basis for simulation design and implementation that can improve consistency in simulated clinical experiences and strengthen research into their effectiveness (Jeffries, 2005). Situated cognition is a framework for learning that combines activity, concept, and culture within educational practice and supports application of learning (Brown, Collins, Duguid, 1989). The purpose of this paper is to introduce the combination of NLN/Jeffries Simulation Framework and situated cognition as a conceptual framework for simulation design and application of learning. This combination will add depth as a conceptual framework for present and future studies.

\section{NLN/Jeffries Simulation Framework}

The NLN/Jeffries Simulation Framework is a theoretical framework developed to organize the variables used in research to measure learner outcomes in nursing education 
using simulation (Jeffries, 2012) (Figure 1). The framework improves consistency in simulation design and implementation thereby strengthening research (Jeffries, 2005). Researchers have applied the framework in several studies including a national multi-site study on designing models for simulation in nursing education (LaFond \& Vincent, 2012).The framework is comprised of five components - facilitator, participant, educational practices, simulation design characteristics, and outcomes - with each component including specific subconcepts and variables (Jeffries, 2012).

The first component, facilitator, includes demographic variables such as age, gender, ethnicity and area of expertise (Jones, Reese, \& Shelton, 2014). The role of the facilitator is observer and supporter of learning in the simulation and debriefing (Jeffries, 2005). Those in this role need to have expertise in running simulations, confidence in teaching, and knowing when to wait and when to provide cues (environment and patient hints that provide direction during the simulation) to support the learning (Jeffries, 2005; Jones et al., 2014).

The participant component includes program, level, and age as its variables (Jeffries, 2012). Gender, culture/ethnicity, type of learner, and interprofessional practice more clearly identify each participant (Durham, Cato, \& Lasater, 2014). Attributes of the participant may include motivation, anxiety, preparation, and prior knowledge (Durham et al., 2014).The participants should actively participate, be engaged, and be self-directed (Jeffries, 2005). They can be team members or individuals participating in collaborative learning but should always feel they are in a safe environment (Durham et al., 2014).

Jeffries (2005) based the educational practices component on Chickering and Gamson's (1987) seven principles of undergraduate education: active learning, feedback, 
student faculty interaction, collaboration, high expectations, diverse learning, and time on task. Active learning engages students in the learning process while the interaction between student and faculty adds depth to the learning and affects student confidence (Hallmark, Thomas, \& Gantt, 2014). Learning is enhanced when objectives are clear and students work collaboratively in teams of the same learning level (Hallmark et al., 2014). Students learn to improve time spent on nursing tasks as they work through simulations. Students and faculty achieve high expectations with well-designed simulations (Hallmark et al., 2014). Feedback on student performance during the simulation assesses knowledge and competence, which is different than debriefing (Hallmark et al., 2014). Debriefing can identify student attitudes with the simulation experience. When students and faculty are of a different gender, race, or ethnicity, and have different backgrounds in patient care, the participants in simulation experience a more diverse learning experience (Hallmark et al., 2014).

Simulation design characteristics include objectives, fidelity, problem solving, student support (cues), and debriefing (Jeffries, 2012). The framework encourages faculty and researchers to address the simulation objectives and develop learner appropriate interactive simulations (Jeffries, 2005). Clearly written objectives are shared with the participants to provide learners with direction (Groom, Henderson, \& Sittner, 2014). Fidelity is the amount of realism in the scenario and includes the level of technology (high, moderate, or low) of the simulator or the use of an actor portraying a patient (Jeffries, 2007). The amount and type of psychomotor skills and expected critical thinking determine the complexity of the simulation or the level of problem solving (Groom et al., 2014). Student support, changed from cues in the original framework, 
includes patient responses and data, orientation of students and faculty to the simulator, and guidance (vague to obvious hints from faculty) throughout the simulation (Jeffries, 2007; Groom et al., 2014). Debriefing is the reflective process following the simulation (Jeffries, 2005). Students share level of confidence and satisfaction with the experience while self-evaluating their attainment of the simulation objectives. This differs from feedback (Hallmark et al., 2014), which can occur during debriefing and includes faculty and peer verification or correction of the psychomotor skills and critical judgment used in the simulation.

Student outcomes include knowledge, skill performance, learner satisfaction, critical thinking, and self-confidence (Jeffries, 2012). The variables are measureable but concerns remain with the reliability and validity of the instruments and the use of selfreport (O’Donnell, Decker, Howard, Levett-Jones, \& Miller, 2014). Research is still needed to determine issues such as appropriate time to conduct simulation in the curriculum, amount of practice needed for skill development, and the transfer of learning and influence on patient care (ODonnell, et al., 2014).

The NLN/Jeffries Simulation Framework aids simulation designers, facilitators, and researchers in improving consistency with design and implementation. The addition of the situated cognition learning framework to the NLN/Jeffries Simulation Framework will further define the active learning strategy. Insert Figure 1. NLN/Jeffries Simulation Framework

\section{Situated Cognition}

Brown et al (1989) framework of situated cognition further defines educational practices in an active learning environment and sets the stage for research on transfer of 
learning in nursing education using simulation. According to Brown, Collins, and Duguid (1989), learning must involve activity, concept, and culture and mirror how the knowledge is used in real situations. Students can manipulate definitions and concepts to show understanding but not be able to apply them. They may be able to pass exams but not use the knowledge in practice. Brown et al. (1989) use the following analogy: students know what a hoof tool is by definition and that a limping horse may require the rider to use the tool to remove a stone from its hoof by definition, but does the student know how to use the tool on the horse's hoof. When learners are able to observe and practice a behavior in a specific situation, actually use the tool on the horse, they absorb the words and actions of the behavior and imitate it until they internalize the behaviors and use them appropriately and consistently. Brown et al. (1989) further stipulate students need to use these tools in activities that are authentic which they define as "ordinary practices of the culture" (p 34). When this exposure occurs, "knowing and doing are interlocked and inseparable" (p 35).

In applying the three main ideas of situated cognition (activity, concepts, and culture) to nursing education, the learning environment can be a simulated hospital room or patient's home whereby students provide care to the patient (actor or mannequin) using the nursing process to problem solve and apply new concepts in different situations. The culture is the profession of nursing and the activity is provision of care in the simulation. The learner accepts the cues from the environment, its culture and the activity to gain knowledge, which is transformed with each new situation (Paige \& Daley, 2009). Faculty led simulations focus on students learning of specific concepts in different 
scenarios increasing their potential for application and transfer of learning to the traditional nursing care settings.

Herrington and Oliver (2000), following a review of the literature on situated learning, identified nine guidelines for use in developing situated learning environments:

1) Provide authentic context that reflect the way the knowledge will be used in real-life

The simulated activities reflect the knowledge, skill and attitudes of the profession of nursing.

2) Provide authentic activities

The simulations follow the nursing process at the level of student leaning and include enough resources that students can apply the learning to real life situations.

3) Provide access to expert performances and the modeling process This may include a videotaped simulation and orientation to the lab to model student expectations. Faculty will support student learning through their participation in the implementation of the simulations.

4) Provide multiple roles and perspectives

5) Support collaborative construction of knowledge

Design simulations with enough time for collaborations and completion of objectives

6) Promote reflection to enable abstractions to be formed

Debriefing immediately after the simulation activities

7) Promote reflection to enable tacit knowledge to be made explicit 
Debriefing immediately after the simulation activities

8) Provide coaching by the teacher at critical times, and scaffolding and fading of teacher support

This introduces the student faculty relationship within simulation. Student support, provided by cues from faculty and staff, may fluctuate during the process as students respond

9) Provide for integrated assessment of learning within the tasks

This addresses assessment of learning and the need to use valid and reliable measures on several gauges of learning.

The authors identify the need for role modeling by experts and interactions with those of varying learner levels. Researchers are beginning to look at the influence of role modeling and the effects of collaboration with other disciplines on student outcomes in the use of simulation in nursing education (Dillon, Noble, \& Kaplan, 2009; Johnson, Lasater, Hodson-Carlton, Siktberg, Sideras, \& Dillard, 2012: Lasater, Johnson, Ravert, \& Rink, 2014). The authors' surmised following their review and development of the guidelines that "useable knowledge is best gained" in situated learning environments (Herrington \& Oliver, 2000, p 3). Therefore, a well designed and implemented simulation incorporating the provided guidelines could improve student knowledge on the concepts identified in the nursing simulation.

Situated cognition (Brown et al., 1989) has been used as a framework by researchers in nursing simulation. Monti, Wren, Haas, and Lupien (1998) applied the framework to the implementation of human patient simulators in their nurse anesthetist program courses and found difficult concepts and infrequently seen situations were better 
understood by students when using the simulator. Wyrostock, Hoffart, Kelly, and Ryba (2014) explored the application of situated cognition to student learning in their international end of life simulation using a standardized patient portraying dying, death, and post mortem care. Students were debriefed immediately following the scenario and one week later; comments indicated increased self-awareness of patient care at end of life and self-efficacy related to future ability to provide this type of care (Wyrostek et al., 2014). The researchers concluded that situated cognition was useful in the "process of constructing their knowledge and understanding of how to effectively provide EOL patient/family-centered care (Wyrostek et al., 2014, p e222). Although the results of this prior work indicate that students feel more confident after participating in a simulation and can apply concepts within the simulation they do not address simulation design or explore the transfer of the learning to a new situation.

\section{Merging the Models}

Combining situated cognition with the NLN/Jeffries Simulation Framework may provide new direction on application of learning to new situations and transfer of learning from the simulated to the clinical setting (Figure 2). Placing the NLN/Jeffries framework within the framework of situated cognition emphasizes that the concepts, the activity and the culture of nursing influence all aspects of simulation design. In authentic learning environments, student actions in response to their prior learning acknowledge their implied understanding (Brown et al., 1989). In each new simulation, their new knowledge and implied understanding is applied to the new scenario. Strong simulation design based on the five constructs and related variables of the NLN/Jeffries Simulation Framework and the guidelines for situated learning environments developed from situated cognition 
may increase opportunities for transfer of student learning from one scenario to another through transformation of learning. The combination of the frameworks and strong simulation design may be the bridge for future research in application of learning to new situations and transfer of learning from simulation to the clinical setting.

The use of simulation in nursing education teaches students to critically think or problem solve in a faculty monitored safe environment. Students are given a patient situation and work as a team in a simulated health care setting to identify nursing procedures and processes, and then implement those that will achieve the best outcome for the patient. Simulation scenarios differ on the needs of the patient (cues), the environment, and the responses of the students at differing levels. Faculty guide private discussions with the students in debriefing following the simulation. This process allows students to think through responses and receive feedback to further their understanding of the concepts and how they may apply this new knowledge to another simulation situation. An Example of Simulation based on the Merged Models

To investigate application of learning from simulation to a videotaped clinical encounter, Kelly, Kossman, Astroth, Forneris, \& Dyck (in review) conducted a quasiexperimental study to determine whether simulation in addition to classroom content improved students' knowledge about elders with dementia and delirium, skills in assessing these conditions, and attitudes towards elders with these diagnoses and learning experiences. The simulation experience was developed using the combination NLN/Jeffries Framework and situated cognition as guidelines for design and implementation, the NLN developed Judy Jones scenario and a videotaped clinical encounter to measure application of learning. Following the NLN/Jeffries Simulation 
Framework as a guide, we first identified participants, facilitators, and educational practices including active participation, diverse learning, high expectations, collaboration, and feedback (Jeffries, 2012). Our participants were senior level undergraduate nursing students between 21 and 22 years of age in our baccalaureate program; the facilitator was a clinical expert in the care of older adults and simulation. The students actively participated in the simulation through communication with the standardized patient and family member and implementation of the nursing process. Diverse learning styles were supported through the simulated hospital room, communications and assessments of the standardized patient, and supplies such as an incentive spirometer in the room. Collaboration was encouraged between the care team and family member. Expectations were higher for this level of student compared to the beginning nursing student. Debriefing of the experience and feedback occurred at completion of the simulation.

Next, we identified the simulation design characteristics including objectives, fidelity, problem solving, student support, and debriefing (Jeffries, 2012). Our objectives included patient safety and completion of the Confusion Assessment Method (CAM) to identify delirium. Actors portrayed the patient and family members in a simulated hospital room with supplies to mimic an authentic clinical setting for fidelity. Students were expected to problem solve through the challenges faced in care of the patient with delirium superimposed on dementia and were supported with cues such as asking about the incentive spirometer. Student outcomes in the framework include learning, skill performance, learner satisfaction, critical thinking, and self-confidence (Jeffries, 2012). We measured student learning (knowledge), skill performance and critical thinking (completion of the CAM following a videotaped encounter), and satisfaction and self- 
confidence (attitudes related to the learning experience and care of patients with dementia and delirium).

Following Herrington and Oliver's (2000) guidelines for situated learning environments (authenticity, multiple roles, collaboration, coaching, reflection, articulation, and assessment of learning), the simulation portrayed care of the older adult with dementia and required the students to actively participate in the nursing process. The expectation in situated cognition is that the scenario will be ill defined to allow students to use their new concepts (knowledge) from theory in the culture (nursing practice) and apply it to the situation (Brown et al., 1989). To provide authentic context and activities reflecting knowledge related to dementia/delirium nursing care, an older adult portrayed the patient with delirium superimposed on dementia in a simulated hospital room with a grandson at her bedside. The students worked together in the roles of primary nurse, secondary nurse, and observers. The patient, family member, and instructor provided cues to support learning and debriefing immediately after the scenario allowed for knowledge building (articulation) and reflection. Learning was assessed within the study through pretests, posttests, and CAM completion.

We found that students participating in the simulation maintained knowledge and improved attitudes from pretest to posttest one month following the simulation (Kelly et al., in review). To skill performance and application of learning, the students viewed a videotaped simulation of an older adult with delirium superimposed on dementia and completed a CAM on the post test. All students in both control and experimental groups successfully identified delirium on the videotaped simulation using the CAM, thus the 
simulation intervention did not differentiate achievement of this outcome (Kelly et al., 2015).

Application of the merged models guided the development and implementation of the simulated experience. Imbedding the NLN/Jeffries Framework in the situated cognition framework added authenticity and improved application of learning to new situations which then facilitates transfer of learning from one simulation to the clinical setting. The students participating in the study were able to apply concepts learned in coursework to the simulation experience then transform this learning and apply it to the videotaped encounter as evidenced by successful completion of the CAM (Kelly et al., 2015).

More research is needed on application of leaning within simulated settings, transfer of learning from simulation to the clinical setting and the use of the merged situated cognition and NLN/Jeffries Simulation Frameworks as a model to support the application of learning form one situation to another situation. Future research includes exploration of student success with use of valid and reliable assessment measures, such as the CAM, in the clinical setting following a simulation and student responses to cues in the simulated environments and clinical setting. 


\section{REFERENCES}

Brown, J. S., Collins, A., \& Duguid, P. (1989). Situated cognition and the culture of learning. Educational Researcher, 18(1), 32-42.

Chickering, A. W. \& Gamson, Z. F. (1999). Development and Adaptations of the Seven Principles for Good Practice in Undergraduate Education. New Directions for Teaching and Learning, 80, 75-81.

Dillon, P. M., Noble, K.A., \& Kaplan, L. (2009). Simulation as a means to foster collaborative interdisciplinary education. Nursing Education Perspectives, 30(2), 87-90.

Durham, C. F., Cato, M. L., \& Lasater, K. (2014). NLN/Jeffries Simulation Framework state of the science project: Participant construct. Clinical Simulation in Nursing, 10, 363-372.

Groom, J. A., Henderson, D., \& Sittner, B. J. (2014). NLN/Jeffries Simulation Framework state of the science project: Simulation design characteristics. Clinical Simulation in Nursing, 10, 337-344.

Hallmark, B. F., Thomas, C. M., \& Gantt, L. (2014). The educational practices construct of the NLN/Jeffries Simulation Framework: State of the science. Clinical Simulation in Nursing, 10, 345-352.

Hayden, J. K., Smiley, R. A., Alexander, M., Kardong-Edgren, S., \&Jeffries, P. R. (2014). The NCSBN National Simulation Study: A longitudinal, randomized, controlled study replacing clinical hours with simulation in prelicensure nursing education. Journal of Nursing Regulation, 5(2), S1-S64.

Herrington, J., \& Oliver, R. (2000). An instructional design framework for authentic learning environments. Educational Technology Research and Development, $48(3), 23-48$

Ironside, P. M. \& McNelis, A.M. (2010). Clinical Education in prelicensure nursing programs: results from a national survey. Nursing Education Perspectives, 31(4), 264-265. 
Jeffries, P. R. (2005). A Framework for designing, implementing, and evaluating simulations used as teaching strategies in nursing. Nursing Education Perspectives, 26(2), 96-103.

Jeffries, P. R. (2012). Simulation in Nursing Education from Conceptualization to Evaluation $\left(2^{\text {nd }}\right.$ ed.), National League for Nursing: New York.

Johnson, E. A., Lasater, K., Hodson-Carlton, K., Siktberg, L., Sideras, S., \& Dillard, N. (2012). Geriatrics in simulation: Role modeling and clinical judgment effect. Nursing Education Perspectives, 33(3), 176-180.

Jones, A. L., Reese, C. E., \& Shelton, D. P. (2014). NLN/Jeffries Simulation Framework state of the science project: The teacher construct. Clinical Simulation in Nursing, $10,353-362$.

Kelly, S. A., Kossman, S. P., Astroth, K. S., Forneris, S. G., \& Dyck, M. J. (in review). Does simulation improve nursing student knowledge, skills, and attitudes in identifying delirium superimposed on dementia, Manuscript submitted for publication.

LaFond, C.M., \& Vincent, C. V. (2012). A critique of the National League for Nursing/Jeffries Framework. Journal of Advanced Nursing, 69(2), 465-480.

Lasater, K., Johnson, E. A., Ravert, P., \& Rink, D. (2014). Role modeling clinical judgment for an unfolding older adult simulation. Journal of Nursing Education, 53(5), 257-264.

Monti, E. J., Wren, K., Haas, R., \& Lupien, A. E. (1998). The use of an anesthesia simulator in graduate and undergraduate education, CRNA: The Clinical Forum for Nurse Anesthetists, 9(2), 59-66.

O’Donnell, J. M., Decker, S., Howard, V., Levett-Jones, T., Miller, C.W. (2014). NLN/Jeffries Simulation Framework state of the science project: Simulation learning outcomes. Clinical Simulation in Nursing, 10, 373-382.

Paige, J., \& Daley, B. J. (20090. Situated cognition: A learning framework to support and guide high-fidelity simulation. Clinical Simulation in Nursing, 5(3), e97-e103.

Wyrostok, L. J., Hoffart, J., Kelly, I., \& Ryba, K. (2014). Situated cognition as a learning framework for international end of life simulation. Clinical Simulation in Nursing, 10, e217-222. 


\section{FIGURES}

\section{The NLN/Jeffries Simulation Framework}

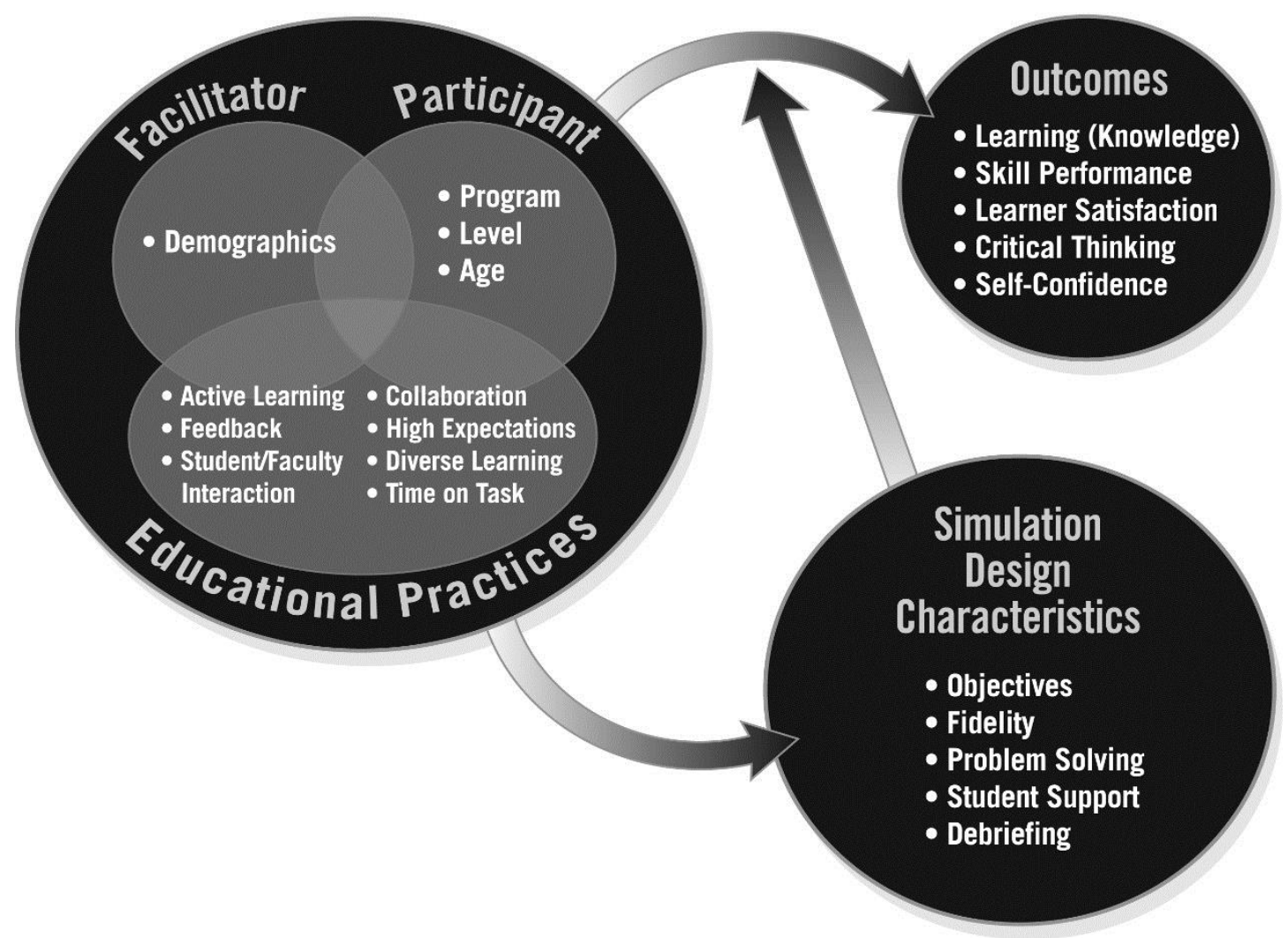

Figure 2. The NLN/Jeffries Simulation Framework.

Copyright permission received from the National League for Nursing 


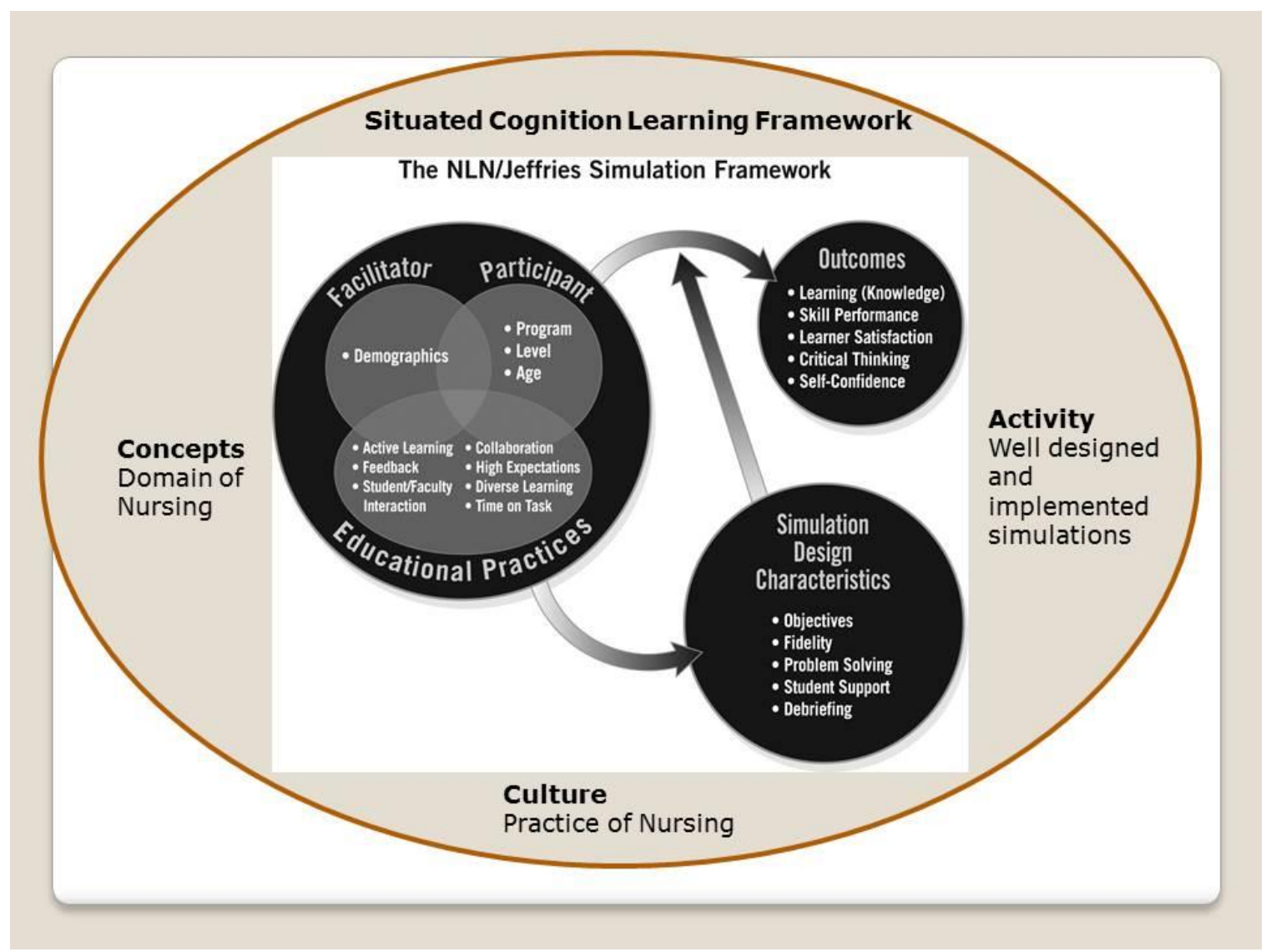

Figure 3. Combination of the Situated Cognition Learning Framework and the NLN/Jeffries Simulation Framework. 


\section{APPENDIX A \\ HIGHLIGHTS, KEY POINTS AND KEY WORDS}

Highlights:

We provided an overview of the NLN/Jeffries Simulation Framework

We provided an overview of the Situated Cognition Learning Framework

We recommend merging the frameworks as guides for research on transfer of learning

Key Points:

NLN/Jeffries Simulation Framework is a guide for simulation design and implementation in nursing education

Situated Cognition Learning Framework posits the need for authentic concepts, activities, and culture to support transfer of learning.

Merging the frameworks in simulation design and implementation guides exploration of application of learning to new situations and transfer of learning from simulation to clinical setting

Key Words:

Situated Cognition, NLN/Jeffries Simulation Framework, Application of learning, Transfer of knowledge, Simulation, Nursing Education 


\title{
CHAPTER III \\ DOES SIMULATION IMPROVE NURSING STUDENT KNOWLEDGE, SKILLS, AND ATTITUDES IN IDENTIFYING DELIRIUM SUPERIMPOSED ON DEMENTIA?
}

Chapter 3 is a manuscript that outlines the method and results of a quasiexperimental study to determine if undergraduate nursing student knowledge, skills, and attitudes improve following a simulation experience. The manuscript will be submitted to Research in Gerontological Nursing for publication.

\begin{abstract}
This quasi-experimental pilot study explored baccalaureate undergraduate nursing student participation in a simulated scenario about nursing care of the patients with dementia and delirium and application of learning. Students in a senior level gerontological nursing course participated in the experimental (didactic content and simulation $n=12$ ) or control (didactic content only $n=14$ ) groups. Results indicated the experimental group had significantly higher post-test knowledge scores than the control group. Both experimental and control groups demonstrated a statistically significant increase in scores from pre- to posttest on the Dementia Attitudes Scale, and the experimental group had a statistically significant increase in scores on the NLN Student Satisfaction and Self Confidence in Learning Instrument. Students in both groups completed the Confusion Assessment Method after viewing a videotaped clinical vignette on posttest and all correctly
\end{abstract}


identified delirium. Well-designed simulation as a pedagogical strategy can benefit geriatric nursing education programs

\section{Introduction}

Delirium occurs in many hospitalized elders, leading to more complicated hospital stays with increased costs (Inouye, 2006), yet nursing staff infrequently assess for delirium and intervene appropriately (Lemiengre et al., 2006; Devlin et al., 2008; Scott, McIlveney, \& Mallice, 2013). The criteria for delirium include a "disturbance in attention and awareness" with a "change in cognition" that "develops over a short period" and "fluctuates during the course of the day" (APA, 2013, p. 596). Nurses are especially challenged to identify delirium in patients who are lethargic, have dementia a "neurocognitive condition with impairment in one or more cognitive areas" (APA, 2013, p. 602), are aged 80 or older or are dually sensory impaired, i.e. both vision and hearing (Cacchione, Culp, Dyck, Laing, 2003; Inouye, Foreman, Mion, Katz, \& Cooney, 2001). Early detection of delirium superimposed on dementia in hospitalized older adults is difficult but necessary for good nursing care (Fick, Hodo, Lawrence, Inouye, 2007). Additional education on assessment of patients with delirium superimposed on dementia is warranted (Lemiengre et al., 2006; Fick et al., 2007; Oligario, Buch, Piscotty, 2015).

Simulation, a pedagogical approach used in nursing education, gives students the opportunity to mimic real life nursing in a controlled environment and move from novice to expert (INACSL, 2013; Jeffries, 2005). According to the International Nursing Association for Clinical Simulation and Learning (INACSL) Standards of Best Practice, a simulation based learning experience includes 
an array of structured activities that represent actual or potential situations in education and practice and allow participants to develop or enhance knowledge, skills, and attitudes or analyze and respond to realistic situations in a simulated environment or through an unfolding case study" (Pilcher et al., cited in INACSL, 2013, p S9).

Prior research indicates practicing nurses and nursing students gain knowledge and improve skills and attitudes in care of the older adult after participation in simulated experiences (Johnson et al., 2012; Landry et al., 2006; Lasater, Johnson, Ravert, \& Rink, 2014; Mager, Lange, Greiner, \& Saracino, 2012; Merchant, 2012; Palmer et al., 2008; Paquette, Bull, Wilson, \& Dreyfus, 2010; Sheets \& Ganley, 2011). A simulated experience for undergraduate nursing students involving care of the hospitalized patient with delirium superimposed on dementia may improve knowledge, skills, and attitudes. However, not enough is known about whether the knowledge, skills and attitudes attained with simulation are transferred to the clinical setting and ultimately improve patient outcomes. The use of a simulated scenario involving care of the hospitalized patient with delirium superimposed on dementia in an undergraduate gerontological nursing course may increase knowledge, improve skills, and change attitudes of undergraduate students in a baccalaureate program. Improving knowledge, skills and attitudes of nurses may subsequently improve nursing care of the hospitalized patient with dementia.

\section{Purpose and Aims}

The purpose of this pilot study is to determine whether undergraduate nursing students increase knowledge and improve skills and attitudes about nursing care of the 
patient with dementia and delirium after participation in a simulated scenario, and can later apply this knowledge and skills to a videotaped clinical encounter.

Specific aims of this study are to determine if 1) a simulation intervention for $4^{\text {th }}$ year undergraduate nursing students increases knowledge, and improves skills and attitudes related to care of the hospitalized patient with dementia and delirium, and 2) the increased knowledge and improved skills and attitudes are retained and correctly applied to a videotaped clinical encounter. The research hypotheses are that undergraduate nursing students in a last semester Gerontological Nursing course who complete required course assignments on dementia and participate in an educational intervention using simulation to care for the hospitalized patient with dementia and delirium (experimental group) will have improved knowledge, skills, and attitudes related to care of the hospitalized patient with dementia and delirium compared to students who only completed the required course assignments on dementia and delirium (control group). In addition, the experimental group students will better retain and apply the improved knowledge, skills, and attitudes in response to a videotaped clinical encounter compared to the control group students.

\section{Framework}

The National League for Nursing (NLN)/Jeffries Framework for Simulation, formerly known as the Nursing Education Simulation Framework, is a conceptual framework that identifies important variables and the relationships among them, thus guiding simulation design, implementation and research evaluating the impact on student learning (Jeffries, 2012). The concepts of the framework include teacher factors (demographics), student factors (program, level, and age), educational practices that need 
to be incorporated into the instruction (active learning, feedback, student faculty interaction, and collaboration), simulation design characteristics (objectives, fidelity, problem solving, student support, and debriefing), and expected student outcomes (knowledge, skill performance, learner satisfaction, critical thinking, and selfconfidence)(Jeffries, 2005). The framework includes Chickering and Gamson's (1987) seven principles of undergraduate education and is based on constructivism (new knowledge gained through experience), sociocultural (interactive learning) and learner centered (instructor guided) learning theories (LaFond \& Vincent, 2012). The framework has been used in several research studies including a national multi-site study on designing models used in simulation in nursing education (LaFond \& Vincent, 2012).

Brown's theory of situated cognition (1989), another learning theory that has been used in research on the use of simulation in nursing education (Monti, Wren, Haas, and Lupien, 1998; Wyrostock, Hoffart, Kelly, and Ryba, 2014), provides additional theoretical concepts. According to Brown, Collins, and Duguid (1989) effective learning must involve activity, concept, and culture. Without these, students may manipulate definitions and concepts to show understanding but not be able to apply them; they may be able to pass exams but not use the knowledge in practice. Brown et al. (1989) stipulate that students "need to be exposed to the use of a domain's conceptual tools in authentic activity" and "authentic activities then, are most simply defined as the ordinary practices of the culture" ( $\mathrm{p}$ 34). Students' knowledge and actions become linked and inseparable when this occurs (Brown et al., 1989). Simulation with scenarios based on the culture of nursing (roles and expectations) is an authentic activity in which students apply concepts 
learned in theory. Students may then take this new knowledge and apply it to each new situation ensuring continued learning (Paige \& Daley, 2009).

The NLN/Jeffries Simulation Framework guides the development of simulation activities by addressing the simulation design characteristics (objectives, fidelity, problem solving, student support, and debriefing).These characteristics assist faculty in determining objectives based on concepts previously learned, realism of the simulated environment, and important cues or hints provided to the student during the simulation. Faculty uses the environment and cues to guide learning as what students see, feel, and interpret within the environment contributes to how they will respond and subsequently learn (Brown et al., 1989). Situated cognition addresses the process of learning through authentic activity and the application of this learning to new situations after the simulation. The combination of the NLN/Jeffries Simulation Framework and Situated Cognition in this study guides how new knowledge and improved skills and attitudes from the simulation are applied to the videotaped clinical encounter. Figure 1 presents the combination of Situated Cognition and the NLN/Jeffries Simulation Framework. In this study, we used an NLN developed scenario that follows the constructs of the NLN/Jeffries Simulation Framework and the additional aspects of the situated cognition framework, concepts, activity and culture, that influence application of learning.

\section{Method}

\section{Research Design}

A quasi- experimental design was used to determine if undergraduate nursing students' participation in a simulated clinical experience involving a hospitalized older adult with dementia and delirium in addition to didactic course content in these areas 
results in a difference in students' knowledge, skills and attitude and their subsequent application to the videotaped clinical encounter. The independent variable of the study is type of instruction - didactic and simulation. Both control and experimental groups received didactic content in an online classroom setting. The experimental group additionally participated in a simulated clinical experience with an actor portraying an older hospitalized adult with dementia and delirium. The dependent variables of the study include knowledge, skills, attitude and application of these to the videotaped clinical encounter. This is a pilot study to test feasibility of the design.

\section{Sample and Setting}

The University Institutional Review Board approved the study and human subject protection. All undergraduate nursing students at a Midwestern university enrolled in an online Gerontological Nursing course during their final semester were invited to participate in the study. In the Gerontological Nursing theory course, students learn nursing care of the older adult including complications associated with dementia and delirium. Inclusion criterion was enrollment in the Spring 2015 section of the Gerontological Nursing course with no exclusion criteria. Recruitment of students occurred by posting study information in an announcement on the course's internet site which is also emailed to each student. Additionally, students were recruited in person at the end of a class meeting of their Leadership/Management course, in which the students are co-enrolled. A convenience sample of 36 students consented to participate. The original plan, to randomly allocate students to the experimental and control groups, was modified due to very slow recruitment. Thus, the first 16 volunteers comprised the experimental group and the remainder of the students were in the control group $(\mathrm{N}=20)$. 
Only 16 students were placed in the experimental group to allow the Primary Investigator to observe a maximum of four groups of four students per group and complete the simulation scenario during one day scheduled in the simulation lab. This improved treatment fidelity by using the same standardized patient actor and the same evaluator for each group of students.

\section{Variables and Instruments}

Table 1 presents the dependent variables with an operational definition and instruments used to measure the variable. A more complete discussion of instruments follows.

Knowledge. Eighteen questions related to care of the patient with dementia and/or delirium taken from the Gerontological Nursing course Exam 1 to measure pre and post intervention knowledge. The following is a sample question from the exam: Mrs. Johnson, a 74 year old woman, was admitted to the nursing home yesterday for rehabilitation following a 5 day hospital stay for a fractured hip and repair. Prior to her hospital stay, she lived alone in her home; her arthritis and hypertension were controlled. Today she has difficulty following directions in therapy. As part of your assessment, you complete a MMSE, a CAM, a clock drawing test, and a GDS. Which test is most appropriate considering your assessment of Mrs. Johnson? Student options: a. MMSE, b. CAM, c. Clock drawing test, d. GDS.

The instructor-developed multiple choice exam was used in previous semesters with content validity established through expert review and Kuder Richardson (KR20). The exam reliability coefficient for the previous semester was 0.5 . 
Skills. Student skills were measured by appropriate assessment of a patient with dementia and correct identification of delirium through use of the Confusion Assessment Method [CAM]. The CAM is an instrument used by healthcare providers and researchers to identify delirium in the practice setting (Inouye et al., 1990). The CAM contains nine assessment questions that relate to four features associated with determining delirium over cognitive impairment; the four features include: 1) acute onset or fluctuating course; 2) inattention; 3) disorganized thinking; and, 4) altered level of consciousness (Inouye et al., 1990). The patient must have features 1 and 2 plus either 3 or 4 to have a diagnosis of delirium (Inouye et al., 1990). The CAM has "demonstrated sensitivities from 90\% to $95 \%$, positive predictive accuracy of $91 \%$ to $94 \%$, negative predictive accuracy of $90 \%$ to $100 \%$ and interrater reliability ranging from 0.81 to 1.00 " (Wei, Fearing, Sternberg \& Inouye, 2008, p 823). The instrument is available for use through the Hartford Institute for Geriatric Nursing.

Student completion of the CAM while observing a videotaped clinical encounter of a nurse interacting with a hospitalized elder patient who has delirium superimposed on dementia measured application of learning.

Attitudes. The Student Satisfaction and Self-Confidence in Learning Instrument measured student satisfaction with the learning, and consisted of 13 items using a five point Likert scale (NLN, 2015). Each item was rated from 1(strongly disagree with the statement) to 5 (strongly agree with the statement). The instrument was adapted with the term simulation removed to determine self-confidence differences between groups. Reliability testing using Cronbach's alpha reported 0.94 for satisfaction and 0.87 for selfconfidence (NLN). The NLN granted permission to use the instrument. 
In addition to the NLN instrument, The Dementia Attitudes Scale (DAS) measures student attitudes related to care of the patient with dementia (O'Connor \& McFadden, 2010). This instrument consists of 20 items using a seven point Likert scale. Each item was rated from 1 (strongly disagree) to 7 (strongly agree). The initial development Cronbach's alpha was reported at 0.83 with undergraduate psychology students (O'Connor \& McFadden, 2010). The same scale was used with nursing students in Malta and the researchers reported a Cronbach's alpha of 0.82 (Scerri \& Scerri, 2012).

\section{Procedure}

The experimental and control groups participated in course activities of the Gerontology course.

Pretest: All students completed the course's first exam during a scheduled exam date and time, which covered material on nursing care of the patient with delirium and/or dementia. In addition, they completed the NLN Student Satisfaction and Self-Confidence in Learning Instrument (NLN, 2015) and the Dementia Attitudes Scale (O'Connor \& McFadden, 2010) in an online survey format.

Experimental intervention: Following completion of Exam 1, students in the experimental group presented to the nursing simulation lab on the same scheduled day at a designated time. These students participate in simulations throughout the curriculum and are familiar with the scenario format of the study site's simulation lab. Groups of four students (role playing a primary and secondary nurse and two observers) completed the Judy Jones scenario described below, with the same actor (Standardized Patient) portraying Judy Jones. The simulation scenario ran in one of the simulated patient rooms wired for audio and video capture within the nursing simulation lab. The evaluator 
observed the students participating in the scenario through video feed and listened to the actor/student exchange through headphones. The Creighton Simulation Evaluation Instrument (CSEI) tailored to this scenario for each group did not measure any of the variables but ensured consistency between groups and guided formative feedback during the 20 minute debriefing session following each simulation (Todd et al., 2008). The instrument incorporates the American Association of Colleges of Nursing (AACN) Essentials and the Quality and Safety Education for Nurses project (QSEN) objectives to measure student knowledge and skills in areas of Assessment, Communication, Critical Thinking, and Technical Skills (Todd et al., 2008). The evaluation instrument is dichotomous and users circle whether the student did or did not demonstrate completion of the objective or skill (Todd et al., 2008). The CSEI was tailored to assess specific student objectives within the study's simulation scenario and these are highlighted in red ink on the instrument.

The Judy Jones scenario developed by the NLN to simulate nursing care of the patient with delirium superimposed on dementia, is available for use on the NLN website (NLN, 2015). The scenario followed Judy Jones, an 85year old patient admitted to the hospital for pneumonia. Judy has dementia with mild cognitive deficits. Her confusion increases during her stay and the students upon entering the room find Judy looking under the bed for her children. The specific scenario student objectives include conducting a focused assessment, demonstrating use of non-pharmacological methods to manage an agitated patient, using the Confusion Assessment Method (CAM) to evaluate the patient for delirium, and taking measures to ensure Judy's safety within the hospital room environment (NLN, 2015). The scenario challenges students to communicate with 
the person with dementia, provide non-pharmacological care and identify the underlying delirium using the CAM. Correct assessment of Judy Jones using the CAM are findings of an alert female with memory problems and an acute onset of disorientation, perceptual disturbances and inattention that fluctuates during the interview. The presence of features 1 , acute onset and fluctuating course, 2, inattention, and 3, disorganized thinking indicate a diagnosis of delirium. Feature 4, altered level of consciousness was not present as the patient was alert and not vigilant (hyperalert), lethargic, in a stupor, or comatose.

Post-test: Four weeks following the intervention, all study students completed the posttests in an online survey format. The posttest included the same pretest 18 questions from Exam 1 that specifically addressed dementia and delirium knowledge and skills, the NLN Student Satisfaction and Self-Confidence in Learning Instrument, and the Dementia Attitudes Scale.

Application of learning: During the post-test period, all study students watched a video vignette of a nurse assessing a hospitalized older patient who has dementia with superimposed delirium. After viewing the video, students completed an online survey including the CAM tool and two open-ended questions to 1) express personal value of the experience and 2) express self-confidence in the completion of the CAM on a patient with delirium superimposed on dementia to determine application of learning of the attitudes variable

\section{Data Analysis}

Quantitative data were collected and analyzed using IBM SPSS 20.0 to address demographics and the specific aims of the study. To address Aim 1, changes in knowledge and attitudes, we used dependent group t-tests to determine mean differences 
between pre- and posttests of each student and group, and independent group ttests to determine group mean differences between the control group and experimental group and calculated effect sizes. To address Aim 2, use of skills and application of learning, we conducted independent t-tests to determine mean differences between the control group and the experimental group on scores on the CAM. Additionally, we completed a thematic analysis of the two open-ended questions and compared groups.

\section{Results}

Demographically, the final sample was female $(96.2 \%)$, white $(92.3 \%)$ or Hispanic $(7.7 \%)$, and either $21(46.2 \%)$ or 22 years of age $(53.8 \%)$. The majority (69.2\%) indicated knowing or having worked with someone with Alzheimer's disease and related disorders. The final sample was 26, with 12 in the experimental group and 14 in the control group. Of the initial 16 students in the experimental group, 13 students completed the pretests and simulation and 12 students completed the posttests. Of the initial 20 students in the control group, 18 completed the pretests and of those, 14 completed the posttests.

\section{Specific Aim 1}

To test whether a simulation intervention for $4^{\text {th }}$ year undergraduate nursing students increases knowledge and improves attitudes related to care of the hospitalized patient with dementia, we analyzed student pre- and post-test scores on the 18 question knowledge test, the NLN Student Satisfaction and Self-Confidence in Learning Instrument, and the Dementia Attitudes Scale.

The results (Table 2) indicated no significant difference between experimental and control groups on knowledge and attitudes pretests indicating a homogenous sample. 
There was a significant decrease in knowledge scores on the post-test for the control group while the experimental group demonstrated a slight, nonsignificant, rise in knowledge scores. The experimental group had significantly higher post-test knowledge scores than the control group. Both experimental and control groups demonstrated a statistically significant increase in scores from pre- to posttest on the Dementia Attitudes Scale. The experimental group had a statistically significant increase in scores on the NLN Student Satisfaction and Self Confidence in Learning Instrument while the control group showed no significant change.

\section{Specific Aim 2}

To test whether increased knowledge and skills are retained and appropriately applied, students completed the CAM after viewing a video vignette of a hospitalized elder with dementia and superimposed delirium. All students in both groups completed the CAM and correctly identified delirium in the vignette of the hospitalized patient, though they varied in their assessment of the nine clinical features of delirium. The majority of students correctly identified clinical features of acute onset, inattention, disorganized thinking, disorientation, memory impairment, perceptual disturbances, psychomotor agitation and altered sleep-wake cycle. However, most students incorrectly assessed her altered level of consciousness as vigilant (hyperalert) rather than alert (treatment 50\% alert and 50\% vigilant, control group 35.7\% alert and 64.3\% vigilant). Those students identifying the patient as being hyperalert then incorrectly selected CAM diagnostic algorithm Feature 4, altered level of consciousness. Thus even though all students correctly identified delirium using the CAM, many were incorrect on the patient's level of consciousness selection. 
The students completed two open-ended questions: 1) to explain how the online material or simulation did or did not influence their self-confidence with the CAM and 2) to explain any change in attitude toward caring for the patient with delirium superimposed on dementia following the learning experience. Both groups responded similarly to these questions. The students' answers to these open-ended questions were analyzed for common themes which follow.

Improved self-confidence. Overall students in the treatment group indicated improved self-confidence with using the CAM to assess a patient for delirium superimposed on dementia following the learning activities. One student replied "I felt more confident interacting with patients experiencing delirium after the simulation and also felt more confident in diagnosing it. It's always helpful to practice these situations in simulations before experiencing them in the real setting". Another student shared, "It helped build some confidence with the CAM assessment because I have never had the opportunity to perform it on a real patient”.

Additionally, students in the control group also expressed improved selfconfidence after watching the video. One student shared, "Seeing the [video]... did influence my self-confidence with CAM, I felt better after seeing it in action, rather than just reading about it". Another shared, "This [video] made me more confident with the CAM. I was able to see the changes in the patient's mental status and saw how the nurse reacted to the patient's confusion, distractedness, and trying to get out of bed". The results indicate student self-confidence in use of the CAM improves when knowing what to expect. Lasater et al. (2014) also identified this theme in their study on role modeling clinical judgment. 
Respect for challenges in caring for these patients. Student responses indicated no change in attitudes but identified a respect for the challenges in caring for these patients. One student from the control group expressed, "My attitude has not changed. I think taking care of a person with delirium superimposed on dementia would be very difficult". A student from the experimental group shared, "I think that with an increased knowledge on how to care for patients with dementia and a new onset delirium can decrease the negative attitudes towards caring for this population". Challenging care is a common theme found in nursing research related to care of patients with dementia (Moyle et al., 2008)

\section{Discussion}

In this study, we hypothesized improved knowledge, skills, and attitudes related to care of the hospitalized patient with dementia and delirium for the experimental group following a simulation compared to the control group with no simulation. The results indicated a significant difference in knowledge and attitude scores on posttest between the experimental and control groups, but we found no difference between groups on skills or application of learning as measured by the CAM. The experimental group maintained their knowledge of delirium and dementia, showing a small, nonsignificant gain at posttest. In contrast, the control group scored significantly lower on the knowledge post-test, indicating that they did not retain this knowledge. A possible explanation for this lower knowledge score is the passage of time between learning this content in class and the posttests. It is possible that the simulation may have counteracted this large effect of passage of time on knowledge. The results of this study reflect what others have found 
with knowledge and skill gain following simulation (Forondo et al., 2013; Gates et al., 2012; Palmer et al., 2008 \& Weaver, 2011)

Additionally, the experimental group showed a significant increase in attitude toward learning, both in self-confidence and satisfaction with learning (NLN scale) also with a large effect size, indicating a large influence of the simulation. However, there were no significant differences between groups in attitudes towards people with dementia; students in both groups had a significant increase in attitude scores towards people with dementia on the DAS post-test. Students in the study engaged in clinical experiences during the study period that included care of hospitalized elders. Perhaps the students had enough contact with patients with dementia in this clinical setting to explain the increase in attitude.

The inability to determine application of learning through the use of the CAM on a videotaped encounter may be due to the short length (4 minutes) of the video, which limited the ability to show less dramatic fluctuations in cognition and level of consciousness of the simulation patient. Although, students indicated increased knowledge, satisfaction and improved confidence with the learning. Another potential factor influencing the lack of statistical significance for the CAM completion post video encounter was prior opportunities for students to use the CAM in the clinical settings. Students are in the clinical setting twice a week for four semesters prior to graduation. Conducting the study over time and at different semesters, may determine the best placement for the simulation in this nursing program. While we did not find a difference between groups in skill performance by the CAM, we did find interesting the student selection on alert vs vigilant. Students in both the experimental (50\%) and control 
(64.3\%) groups selected vigilant over alert for the level of consciousness. The patient was alert and sitting in bed while the nurse was present. The patient's attempts to get out of bed may have been seen as hyperalert by the students.

In sum, we found that simulation had a strong impact on knowledge, self confidence and satisfaction with learning even in this small of a sample, but could not detect its influence on student gains in skills and attitudes towards people with dementia. This study supports prior research findings of improvement in knowledge and attitudes, especially self-confidence following the use of simulation as a pedagogical strategy in nursing education (Foronda et al., 2013;Gates et al., 2012; Hallenbeck, 2012; Weaver, 2011). Research supporting evaluation methods to determine improved skills following a High Fidelity Simulation (HFS) is very limited (Knudson et al., 2008; Shapiro et al., 2004) and is non-existent in geriatric nursing education related to care of the patient with dementia and delirium. This study is the first to use the CAM as a measure of skill improvement following simulation.

\section{Limitations}

There are four limitations to this study: a small convenience sample, selection bias, timing of the data collection and intervention, and the researcher as debriefer. We recruited students from a single class of 64 senior baccalaureate nursing students in one College of Nursing. Student recruitment was challenging. The first 16 students volunteering to participate were placed in the treatment group and recruitment continued for several weeks to gather the control group students. Clarification of the pretests and posttests as online surveys resulted in additional students participating, perhaps because of ease in completing the surveys at convenient times and locations. Timing of the pre- 
and posttest surveys and intervention also posed challenges. The intervention was rescheduled due to inclement weather and three volunteers could not attend at the rescheduled time. Post-test surveys were completed near the end of the semester, a time when students are over-committed with additional testing and finishing projects. Several students mentioned inability to participate due to weekend clinical and other course work. Finally, there is a potential confounding effect due to the researcher serving as debriefer. The researcher had prior knowledge of the pre- and post-tests which may have influenced the direction of the debriefing. Paget (2001) discusses the essential role of the facilitator and notes "Many factors contribute to the influence of the debriefer including:

personality, style, knowledge, familiarity, perception of effectiveness, and development of relationship with participants (p. 206). However despite these limitations, we found significant differences in knowledge and attitudes at posttest in groups which were similar at the onset of the study (knowledge pretest: treatment $\mathrm{M}=16.0$ and control $\mathrm{M}=$ 15.9 and attitudes pretests: treatment $M=93.1$ and control $M=94.9$ ).

\section{Conclusion}

The challenges with conducting this study, although influential in our ability to determine statistical significance on the variable skills, inspire additional questions and possibilities for future research. The questions warrant a descriptive study on variables influencing transfer of learning so we may clearly define whether the high fidelity simulation or other outside influences improved knowledge and self-confidence. Lack of significant findings on the transfer of learning to the clinical area suggests further research is needed. Incidental results indicate research may include the effects of using videotaped simulated clinical encounters as role modeling. The use of measurements with 
documented validity and reliability testing and a simulation developed by experts from the NLN and evaluated by several simulation programs across the country, strengthen the opportunities for increased sample size through multi-site collaboration. The use of rigorous design and valid and reliable measurements in future research will strengthen simulation as a pedagogical strategy and benefit geriatric nursing education programs. 


\section{REFERENCES}

American Psychiatric Association (2013). Diagnostic and Statistical Manual of Mental Disorders, Fifth Edition. $5^{\text {th }}$ ed. Washington, DC: American Psychiatric Association.

Brown, J. S., Collins, A., Duguid, P. (1988). Situated cognition and the culture of learning. Institute for Research on Learning, BBN Systems and Technologies Corporation, Report No. 6886, 1-32.

Cacchione, P. Z. , Culp, K., Dyck, M. J., Laing, J. (2003). Risk for acute confusion in sensory-impaired, rural, long-term care elders. Clinical Nursing Research, 12(4), 340-355.

Chickering, A. W. \& Gamson, Z. F. (1999). Development and Adaptations of the Seven Principles for Good Practice in Undergraduate Education. New Directions for Teaching and Learning, 80, 75-81.

Devlin, J. W., Fong, J. J., Howard, E. P., Skrobik, Y., McCoy, N., Yasuda, C., Marshall, J. (2008). Assessment of delirium in the intensive care unit: Nursing practices and perceptions. American Journal of Critical Care, 17(6), 555-565.

Fick, D. M., Hodo, D. M., Lawrence, F. \& Inouye, S. K. (2007). Recognizing delirium superimposed on dementia: Assessing nurses' knowledge using case vignettes. Journal of Gerontological Nursing, 33(2), 40-49.

Foronda, C., Liu, S., Bauman, E. B. (2013). Evaluation of simulation in undergraduate nurse education: An integrative review. Clinical Simulation in Nursing, 10, e409416.

Gates, M. G., Parr, M. B., \& Hughen, J. E. (2012). Enhancing nursing knowledge using high-fidelity simulation. Journal of Nursing Education, 51(1), 9-14.

Hallenbeck, V. J. (2012). Use of high-fidelity simulation for staff education development: A systematic review of the literature. Journal for Nurses in Staff Development, 28(6), 260-269. 
Inouye, S., van Dyck, C., Alessi, C., Balkin, S., Siegal, A. \& Horwitz, R. (1990). Clarifying confusion: The confusion assessment method. Annals of Internal Medicine, 113 (12), 941-948.

Inouye, S. K., Foreman, M. D.,Mion, L. C., Katz, K. H., \& Cooney Jr., L. M. (2001). Nurses' recognition of delirium and its symptoms. Archives of Internal Medicine, 161(20), 2467.

Inouye, S. K. (2006). Delirium in older persons. New England Journal of Medicine, 354(11), 1157-1165.

International Nursing Association for Clinical Simulation and Learning (2013). Standards of best practice: Simulation. Clinical Simulation in Nursing, 9, S1-S32.

Jeffries, P. R. (2005). A Framework for designing, implementing, and evaluating simulations used as teaching strategies in nursing. Nursing Education Perspectives, 26(2), 96-103.

Jeffries, P. R. (2012). Simulation in Nursing Education from Conceptualization to Evaluation $\left(2^{\text {nd }} \mathrm{ed}.\right)$, National League for Nursing: New York.

Johnson, E. A., Lasater, K., Hodson-Carlton, K., Siktberg, L., Sideras, S., \& Dillard, N. (2012). Geriatrics in simulation: Role modeling and clinical judgment effect. Nursing Education Perspectives, 33(3), 176-180.

Knudson, M. M., Khaw, L., Bullard, M. K., Dicker, R., Cohen, M. J., Staudenmayer, K, Krummel, T. (2008). Trauma training in simulation: translating skills from SIM time to real time. Journal of Trauma, 64 (2), 255-64.

LaFond, C.M., \& Vincent, C. V. (2012). A critique of the National League for Nursing/Jeffries Framework. Journal of Advanced Nursing, 69(2), 465-480.

Landry, M., Oberleitner, M. G., Landry, H., \& Borazjani, J. G. (2006). Education and practice collaboration: Using simulation and virtual reality technology to assess continuing nurse competency in the long-term care acute care setting. Journal for Nurses in Staff Development, 22(4), 163-169.

Lasater, K., Johnson, E. A., Ravert, P., \& Rink, D. (2014). Role modeling clinical judgment for an unfolding older adult simulation. Journal of Nursing Education, 53(5), 257-264.

Lemiengre, J., Nelis, T., Joosten, E., Braes, T., Foreman, M., Gastmans, C., \& Milisen, K.(2006). Detection of delirium by bedside nurses using the confusion assessment method. Journal of the American Geriatrics Society, 54, 685-689. 
Mager, D. R., Lange, J. W., Greiner, P. A., \& Saracino, K. H. (2012). Using simulation pedagogy to enhance teamwork and communication in the care of older adults: The ELDER project. The Journal of Continuing Education in Nursing, 43(8), 363-369.

Merchant, D. C. (2012). Does high-fidelity simulation improve clinical outcomes? Journal for Nurses in Staff Development, 28(1), E1-E8.

Monti, E. J., Wren, K., Haas, R., \& Lupien, A. E. (1998). The use of an anesthesia simulator in graduate and undergraduate education, CRNA: The Clinical Forum for Nurse Anesthetists, 9(2), 59-66.

National League for Nursing (2014). Simulation Innovation Resource Center. Retrieved from http://sirc.nln.org/.

O’Connor, M. L. \& McFadden, S. H. (2010). Development and Psychometric Validation of the Dementia Attitudes Scale. International Journal of Alzheimer's Disease, $1-10$.

Oligario, G. C., Buch, C., \& Piscotty, R. (2015). Nurses' assessment of delirium with underlying dementia in end of life care. Journal of Hospice and Palliative Nursing, 17(1), 16-21.

Paget T. (2001). Reflective practice and clinical outcomes: practitioners' views on how reflective practice has influenced their clinical practice. Journal of Clinical Nursing, 10(2), 204-214.

Paige, J., \& Daley, B. J. (20090. Situated cognition: A learning framework to support and guide high-fidelity simulation. Clinical Simulation in Nursing, 5(3), e97-e103.

Palmer, M. H,. Kowlowitz, V., Campbell, J., Carr, C., Dillon, R., Durham, C. F., Rasin, J. (2008). Using clinical simulations in geriatric nursing continuing education. Nursing Outlook, 56, 159-166.

Paquette, M., Bull, M., Wilson, S., \& Dreyfus, L. (2010). A complex elder care simulation using improvisational actors. Nurse Educator, 35(6), 254-258.

Scerri, A. \& Scerri, C. (2013). Nursing students' knowledge and attitudes towards dementia - A questionnaire survey. Nurse Education Today, 33, 962-968.

Scott, P., McIlveney, F., \& Mallice, M.(2012). Implementation of a validated delirium assessment tool in critically ill adults. Intensive and Critical Care Nursing, 29, 96-102. 
Shapiro, M. J., Morey, J. C., Small, S. D., Langford, V., Kaylor, C. J., Jagminas, L., ... Jay, G. D. (2004). Simulation based teamwork training for emergency department staff: Does it improve clinical team performance when added to an existing didactic teamwork curriculum? Quality and Safety in Healthcare, 13(6), 417421.

Sheets, I. W. \& Ganley, B. J. (2011). Integrating simulation into a foundational gerontological nursing course. Journal of Nursing Education, 50(12), 689-692.

Todd, M., Manz, J., Hawkin, K., Parsons, M. \& Hercinger, M. (2008). The development of a quantitative evaluation tool for simulation in nursing education. International Journal of Nursing Education Scholarship, 5(1), Article 41.

Weaver, A. (2011). High-fidelity patient simulation in nursing education: An integrative review. Nursing Education Perspectives, 32(1), 37-40.

Wei, L. A., Fearing, M. A., Sternberg, E. J. \& Inouye, S. K. (2008). The confusion assessment method: A systematic review of current usage. Journal of the American Geriatrics Society, 56, 823-830.

Wyrostok, L. J., Hoffart, J., Kelly, I., \& Ryba, K. (2014). Situated cognition as a learning framework for international end of life simulation. Clinical Simulation in Nursing, 10, e217-222. 


\section{FIGURES}

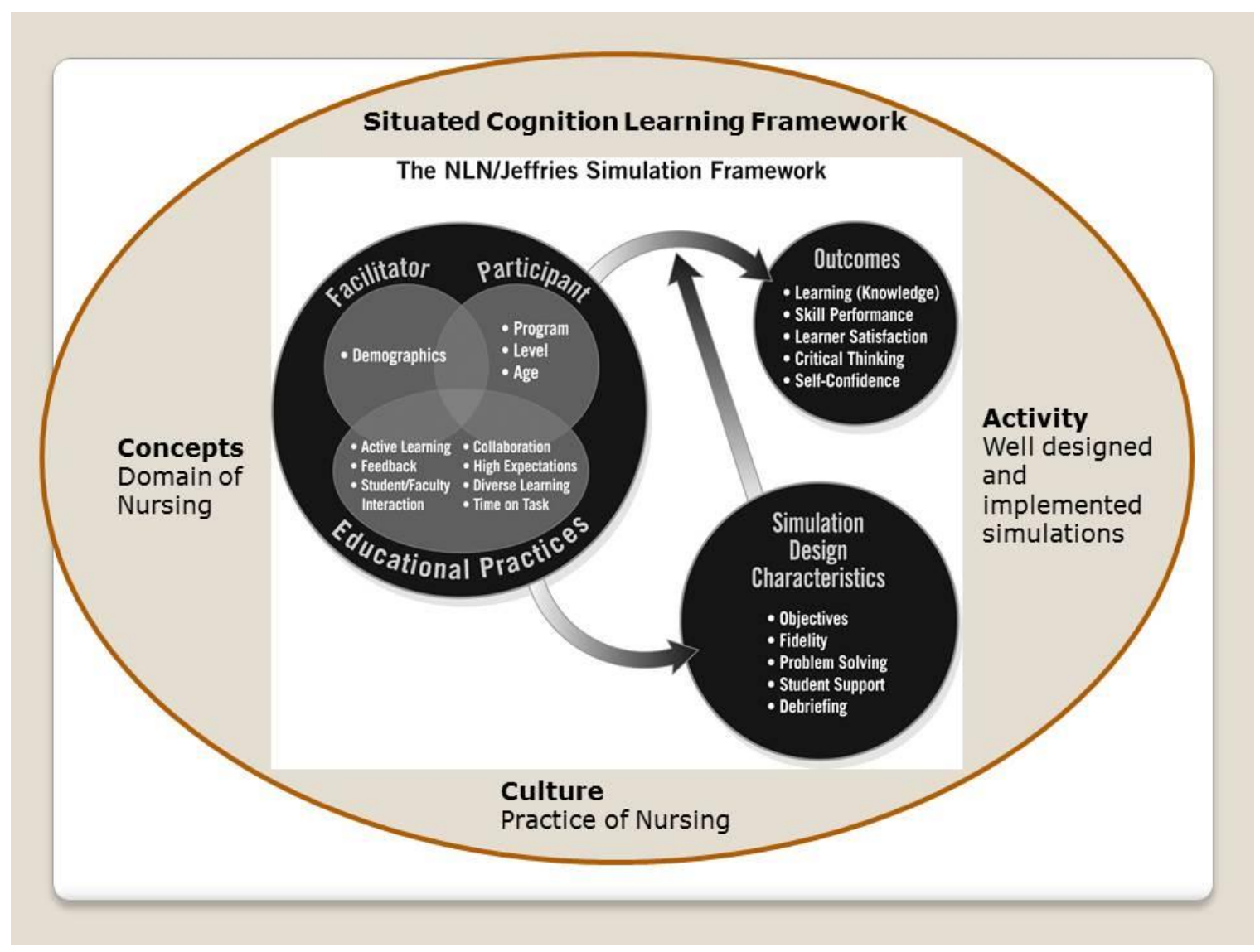

Figure 4. Combination of the Situated Cognition Learning Framework and the NLN/Jeffries Simulation Framework.

Copyright permission for the NLN/Jeffries Simulation Framework received from National League for Nursing 


\section{TABLES}

Table 1

Variables, Operational Definitions, and Instruments

\begin{tabular}{|c|c|c|c|}
\hline Variable & $\begin{array}{c}\text { Operational } \\
\text { Definition }\end{array}$ & Instruments & $\begin{array}{c}\text { Validity and } \\
\text { Reliability }\end{array}$ \\
\hline Knowledge & $\begin{array}{l}\text { Scores on a } \\
\text { knowledge exam } \\
\text { related to care of the } \\
\text { hospitalized patient } \\
\text { with dementia and } \\
\text { delirium }\end{array}$ & $\begin{array}{l}\text { Pretest and posttest } \\
\text { are the same }=18 \\
\text { questions on } \\
\text { delirium/dementia } \\
\text { from the } \\
\text { Gerontology Nursing } \\
\text { Course Exam1 }\end{array}$ & KR $20-0.53$ \\
\hline Skills & $\begin{array}{l}\text { Ability of the } \\
\text { student to perform } \\
\text { an accurate } \\
\text { assessment of } \\
\text { delirium in a patient } \\
\text { with dementia and } \\
\text { delirium }\end{array}$ & $\begin{array}{l}\text { Confusion } \\
\text { Assessment Method } \\
{[\mathrm{CAM}]}\end{array}$ & $\begin{array}{l}\text { Sensitivity - } \\
90 \%-95 \%\end{array}$ \\
\hline Attitudes & $\begin{array}{l}\text { Score on self- } \\
\text { confidence, learning } \\
\text { and attitude scales } \\
\text { related to care of a } \\
\text { patient with } \\
\text { delirium } \\
\text { superimposed on } \\
\text { dementia. }\end{array}$ & $\begin{array}{l}\text { NLN Student } \\
\text { Satisfaction and Self- } \\
\text { Confidence in } \\
\text { Learning Instrument } \\
\\
\text { Dementia Attitudes } \\
\text { Scale (DAS) }\end{array}$ & $\begin{array}{l}\text { NLN Instrument: } \\
\text { Cronbach's } \alpha \text { - } \\
0.94 \text { for } \\
\text { satisfaction and } \\
0.87 \text { for self- } \\
\text { confidence } \\
\text { DAS: Cronbach's } \\
\alpha-0.82 \text { with } \\
\text { nursing students }\end{array}$ \\
\hline
\end{tabular}

Table continued 


\begin{tabular}{|l|l|l|l|}
$\begin{array}{c}\text { Application of } \\
\text { Learning }\end{array}$ & $\begin{array}{l}\text { Ability to apply } \\
\text { knowledge and } \\
\text { skills gained in } \\
\text { classroom and } \\
\text { simulation } \\
\text { experiences to a } \\
\text { new clinical } \\
\text { situation and } \\
\text { positive accurate } \\
\text { completion }\end{array}$ & $\begin{array}{l}\text { CAM accurately } \\
\text { completed while } \\
\text { watching a } \\
\text { videotaped clinical } \\
\text { encounter of } \\
\text { patient/nurse } \\
\text { interaction for a } \\
\text { patient with delirium } \\
\text { superimposed on } \\
\text { dementia }\end{array}$ & $\begin{array}{l}\text { Sensitivity } \\
90 \%-95 \%\end{array}$ \\
& & $\begin{array}{l}\text { Two open-ended } \\
\text { questions about } \\
\text { experience of using } \\
\text { CAM to assess a } \\
\text { patient with delirium } \\
\text { and dementia, } \\
\text { following videotaped } \\
\text { clinical encounter }\end{array}$ & \\
& &
\end{tabular}




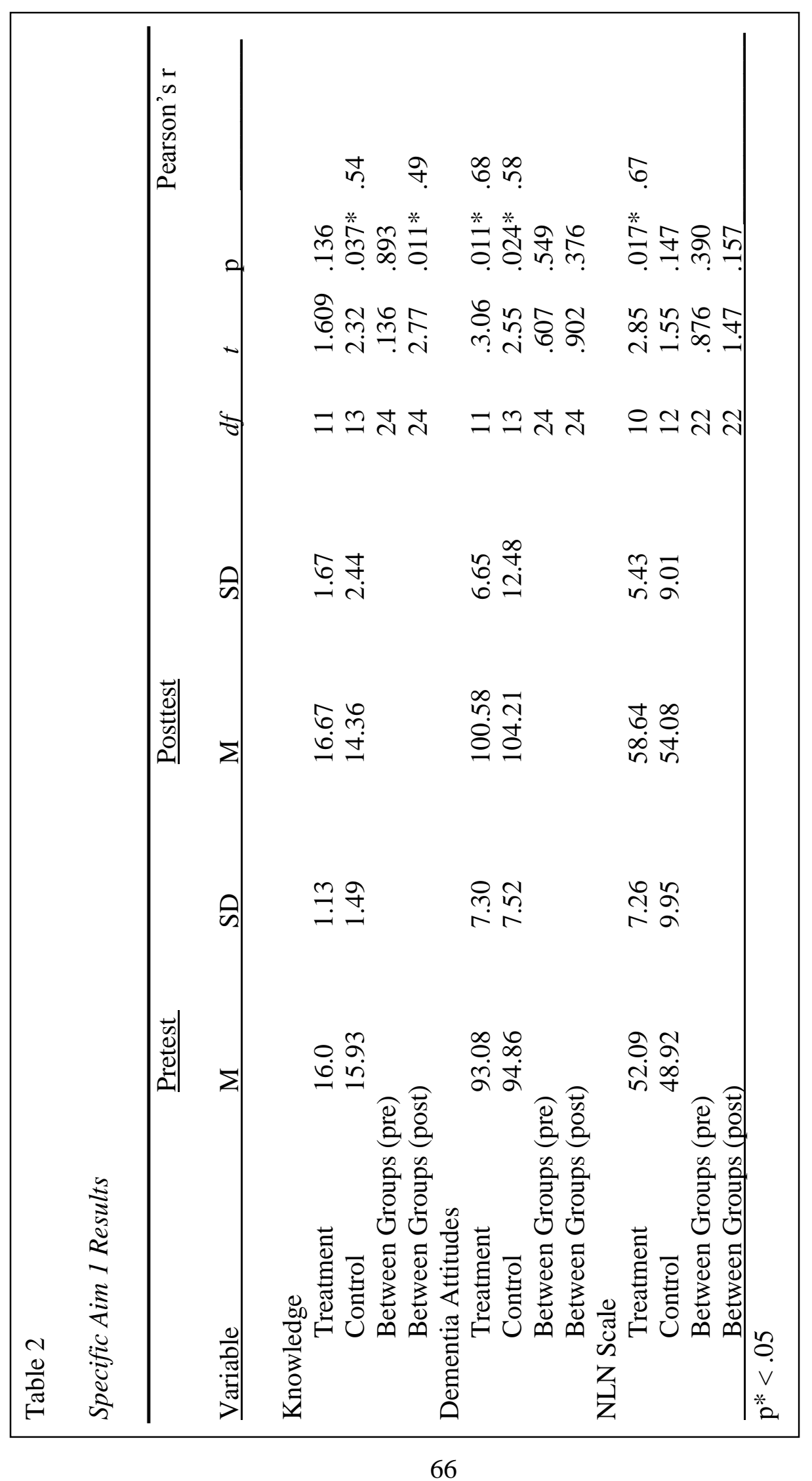

